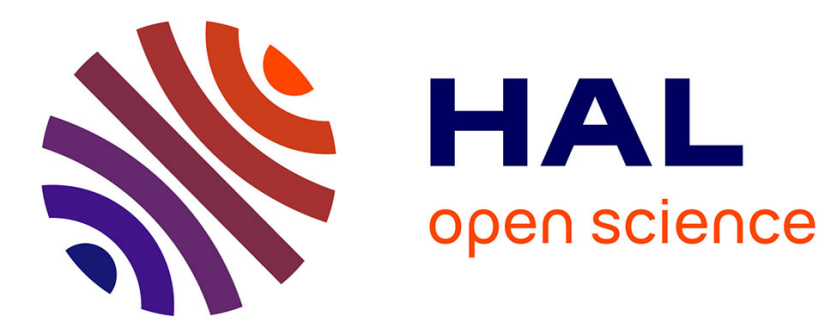

\title{
Capacity Results for the Multicast Cognitive Interference Channel
}

Meryem Benammar, Pablo Piantanida, Shlomo Shamai Shitz

\section{To cite this version:}

Meryem Benammar, Pablo Piantanida, Shlomo Shamai Shitz. Capacity Results for the Multicast Cognitive Interference Channel. IEEE Transactions on Information Theory, 2017, 63 (7), pp.4119 4136. 10.1109/TIT.2017.2702565 . hal-01742445

\section{HAL Id: hal-01742445 \\ https://hal-centralesupelec.archives-ouvertes.fr/hal-01742445}

Submitted on 16 Sep 2020

HAL is a multi-disciplinary open access archive for the deposit and dissemination of scientific research documents, whether they are published or not. The documents may come from teaching and research institutions in France or abroad, or from public or private research centers.
L'archive ouverte pluridisciplinaire HAL, est destinée au dépôt et à la diffusion de documents scientifiques de niveau recherche, publiés ou non, émanant des établissements d'enseignement et de recherche français ou étrangers, des laboratoires publics ou privés. 


\title{
Capacity Results for the Multicast Cognitive Interference Channel
}

\author{
Meryem Benammar, Member, IEEE, Pablo Piantanida, \\ Senior Member, IEEE, and Shlomo Shamai (Shitz), Fellow Member, IEEE
}

\begin{abstract}
The capacity region of the Multicast Cognitive Interference Channel (CIFC) is investigated. This channel consists of two independent transmitters that wish to multicast two different messages, each of them to a different set of users. In addition, one of the transmitters -commonly referred to as the cognitive transmitter- has prior non-causal knowledge of both messages to be transmitted. This scenario combines difficulties and challenges arising in the Interference Channel, the Broadcast Channel and multicasting communications. Our aim concerns the derivation of optimal interference mitigation techniques in such a challenging communication setup. We investigate to this end the multi-primary CIFC and its dual multi-secondary CIFC under various interference regimes as an attempt to build a thorough understanding for the more general setting. It is shown that, for some regimes, well-known coding techniques for the conventional CIFC remain still optimal in the presence of multicasting. While in other regimes, evolved encoding and/or decoding strategies are crucial. A careful use of these coding schemes and new outer bounding techniques allows us to characterize the capacity region for several classes of discrete memoryless and Gaussian channels in different interference regimes.
\end{abstract}

\section{Index Terms}

The material in this paper was partially published in the IEEE Information Theory Workshop, Jerusalem, April 26 to May 1, 2015 and and in the IEEE International Symposium on Information Theory, Hong Kong, June 14-19, 2015. This research was partially supported by the FP7 Network of Excellence in Wireless COMmunications NEWCOM\#.

Meryem Benammar is with the Mathematical and Algorithmic Sciences Lab, France Research Center, Huawei Technologies Co., Ltd (e-mail: meryem.benammar@huawei.com).

Pablo Piantanida is with the Laboratoire des Signaux et Systèmes (L2S UMR 8506) at CentraleSupelec-CNRS-Université Paris-Sud, France (e-mail: pablo.piantanida@centralesupelec.fr).

Shlomo Shamai (Shitz) is with the Department of Electrical Engineering, Technion, Haifa, Israel, (e-mail: sshlomo@ee.technion.ac.il). 
Cognitive interference channel, multiple description coding, multicasting, compound interference channel, interference decoding, interference precoding. 


\section{INTRODUCTION}

The Cognitive Interference Channel (CIFC) was first introduced by Devroye et al. [1] as an interference channel with two sources and two destinations in which one of the sources has full non causal knowledge of both messages to be transmitted, as depicted in Fig. 11. The cognitive source models the secondary transmitter of a cognitive radio environment, which, upon sensing the primary transmitter's message, communicates the secondary message to the secondary user. As such, the secondary source should not create too much interference in the secondary transmission so as not to cause impediment to the primary communication, however, it can also cooperate with the primary source and thus enhance the performances of the primary communication. As it is defined, a CIFC can be regarded as a Broadcast Channel with a helper, i.e., the primary encoder. The helper enhances the transmission of the message $W_{1}$ in the $\mathrm{BC}$ formed by $X_{2}$ and the two destinations $(Y, Z)$, however, it creates more interference at user $Z$ that is interested only in message $W_{2}$. The optimal transmission strategy needs to capture such a tradeoff, and is thus hitherto unknown, however, a few cases denoted as interference regimes are to this day fully understood.

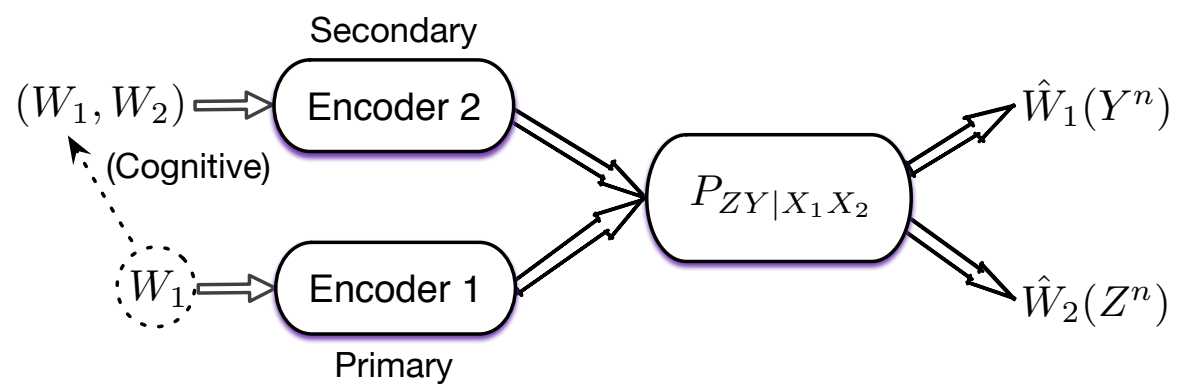

Fig. 1. The Cognitive Interference Channel.

The first capacity result of this setting is due to Maric et. al [2] for the "very strong interference" regime based on an equivalence with the Interference Channel (IFC) with a common message. Later Wu et. al in [3], and independently Jovicic et. al in [4], characterized the capacity region of the "very weak interference" regime. The capacity of the Z-CIFC with a noiseless link was derived by Liu et al. in [5] and the classes of Less-Noisy and More-Capable CIFC were investigated in the works of Vaezi [6], [7]. Lately, Rini et. al proposed in [8] a unifying inner bound that is capacity achieving in all regimes in which capacity is known, through a combination 


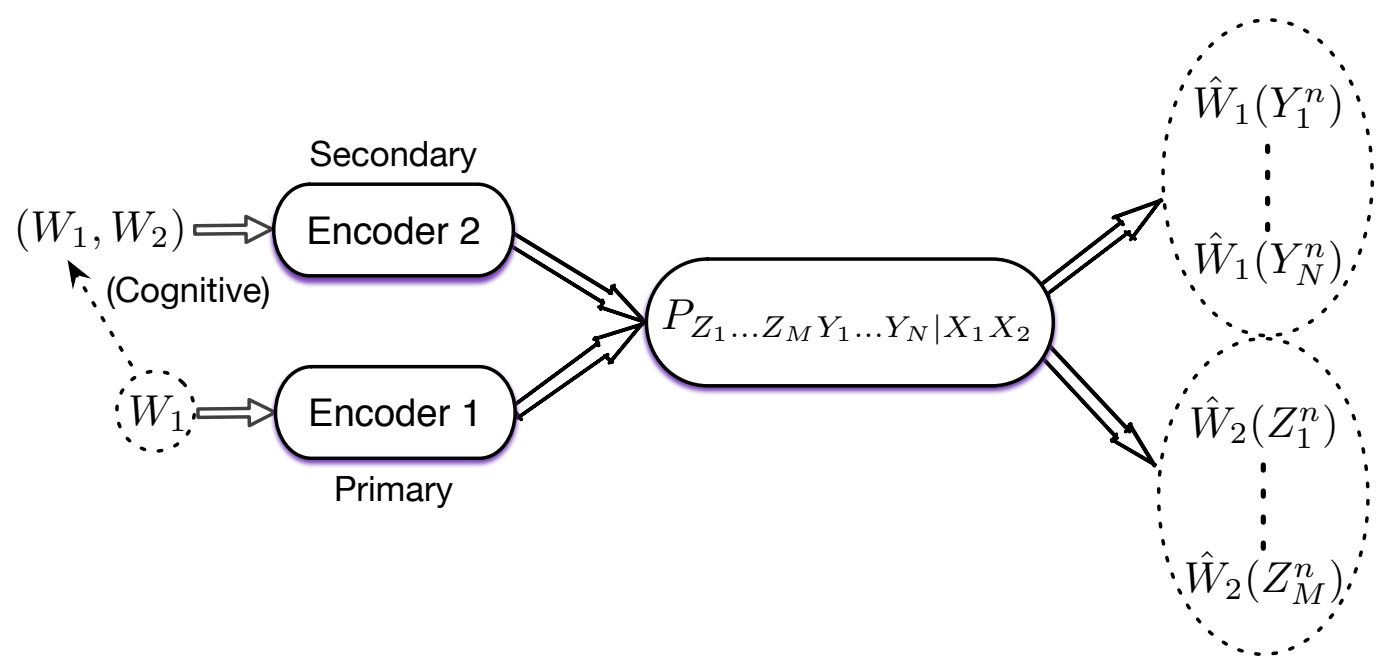

Fig. 2. The Multicast Cognitive Interference Channel.

of known techniques of binning, rate splitting, and superposition coding. They also suggest a new outer bound that alleviates the computational complexity of existing outer bounds which involve auxiliary random variables. Additionally, based on the proposed inner bound, the capacity region of a new regime, denoted as " better cognitive decoding" regime, was derived along with the capacity of the semi-deterministic CIFC.

As for the Gaussian CIFC, the capacity region remains to be fully characterized, yet, some regimes are fully understood: the weak interference capacity region was derived in [3], capacity of the very strong interference was reported in [2] and that of the primary decodes interference regime was found in [9]. The S-CIFC, where interference is experienced only at the primary user's side, was also extensively studied and capacity for the weak interference case was characterized under different regimes by Jiang et al. in [10], Vaezi et al. in [11], and Rini et al. in [9]. Extensions to multiple users were attempted in the rather exhaustive survey of [12] where many messages are to be delivered to many receivers from many transmitters, a subset of which have non-causal knowledge of some messages.

In this paper, we investigate another challenging multi-user scenario where multicasting transmission over a CIFC are combined, as depicted in Fig. 2. The resulting Multicast CIFC is composed of many primary users interested in a same message $W_{1}$ and many secondary receivers similarly interested in a same message $W_{2}$. A possible deployment scenario of this setting consists 
of several users (spectators) in a football stadium wishing to have access to instantaneous replay of the most important actions on their cell phones while being served by two base stations: a primary one destined to the hosting team, and a secondary one intended to serve the guest team.

The aim of this work is to infer optimal interference mitigation techniques, considering this multicast setting, through the understanding of how coding schemes should account for multicasting among both primary and secondary receivers. This task is rendered challenging by both the fact that the capacity region for the general CIFC is not known and that multicasting imposes design efficient interference mitigation techniques that could accommodate interference for many users at once. Indeed, even in basic setups of multicast (or compound) Broadcast Channels, i.e. without the helper, optimal schemes remains still unknown. In particular, we

investigate two dual classes of Multicast CIFC: the Multi-Primary CIFC where we consider only one secondary receiver and design the optimal schemes to serve a set of $N$ primary receivers, and its dual Multi-Secondary CIFC. The two classes of Multicast CIFC bring distinct challenges to face both at the encoding and at the decoding sides. It is shown that under specific interference regimes, some known optimal schemes without multicasting remain optimal even in the presence of multiple users. Whereas for other interference regimes, the introduction of more evolved encoding and decoding strategies is necessary to derive capacity results. These strategies consist in the two ideas of "Interference Decoding" and "Multiple Description coding" [13] whose introduction will turn out to yield either optimality result, for the Multi-Primary CIFC, or a significant improvement over standard encoding techniques in the Multi-Secondary CIFC.

\section{Contributions and outline:}

We start by deriving in Section $I$ an inner bound to the capacity region of the general Mutlicast CIFC which combines optimal coding techniques of the Broadcast Channel (superposition coding and random binning) with rate-splitting for the Interference Channel, and which generalizes Marton's inner bound to the Multicast setting. Then, in the remainder of the work, we evaluate this rate region in both classes of Multi-Primary and Multi-Secondary CIFC in very strong interference and very weak interference and a new regime denoted as mixed very weak/string interference, as well as the Gaussian counterparts of these regimes. In Section III, we investigate the Multi-Primary CIFC and derive the following results:

- The capacity region of the very strong interference regime, 
- The capacity region of the very weak interference regime through a novel outer bound that appears to be necessary to palliate the use of Csiszár \& Körner sum-identity,

- The capacity region of the mixed very weak/strong interference regime while resorting to the idea of Interference Decoding,

- The capacity region of the corresponding Gaussian Multi-Primary CIFC in very strong interference, weak interference, and mixed weak/very strong interference regimes.

In Section IV, we investigate the Multi-Secondary CIFC and characterize, similarly to the Mutli-Primary CIFC, and derive the following results:

- The capacity region of the very strong interference regime,

- The capacity region of the very weak interference regime through a novel outer bound that palliates the use of Csiszár \& Körner sum-identity,

- The capacity region of the mixed very weak/strong interference regime,

- The capacity region of the corresponding Gaussian Multi-Secondary CIFC in very strong interference regime.

It is worth mention that the Gaussian Multi-Secondary CIFC in the weak interference regime appears to be particularly challenging, as shown in the light of the work by Khisti et al. [14]. We address this difficulty that arises from multicasting constraint by sending multiple views -intended to different users- of the same information which resorts to Multiple Description coding. Although in this case the outer and inner bound we derived are not tight, our inner bound strictly outperforms the inner bound where only a common description is used.

In Section V, we elaborate on a peculiar fact in the standard (non-multicast) CIFC which is that the very strong interference and the better cognitive decoding are both included in the very weak interference regime as proved by Vaezi in [15]. We show that this claim does not hold in the considered scenario when we are in the presence of a strict multicast, i.e. $N>1$ or $M>1$.

\section{Notations and conventions}

For any sequence $\left(x_{i}\right)_{i \in \mathbb{N}_{+}}$, notation $x_{k}^{n}$ stands for the collection $\left(x_{k}, x_{k+1}, \ldots, x_{n}\right) . x_{1}^{n}$ is simply denoted by $x^{n}$. Entropy is denoted by $H(\cdot)$, and mutual information by $I(\cdot ; \cdot)$. E resp. $\mathbb{P}$ denote the expectation w.r.t. the generic probability distributed (PD) while the notation $P$ is specific to the probability of a random variable (rv). Let $X$ and $Y$ be random variables, we denote by $P_{X Y}$ (resp. $P_{Y \mid X}$, and $\left.P_{X}\right)$ the joint probability distribution of $(X, Y)$ (resp. conditional 
distribution of $Y$ given $X$, and marginal distribution of $X$ ). $\|\mathcal{X}\|$ stands for the cardinality of the set $\mathcal{X}$. We denote typical and conditional typical sets by $T_{\delta}^{n}(X)$ and $T_{\delta}^{n}\left(Y \mid x^{n}\right)$, respectively (see Appendix A for details). Let $X, Y$ and $Z$ be three random variables on some alphabets with probability distribution $p$. If $p(x \mid y z)=p(x \mid y)$ for each $x, y, z$, then they form a Markov chain, which is denoted by $X \odot Y \odot Z$.

\section{PROBlem DEFINITION AND INNER BOUND}

The discrete memoryless N-Multicast CIFC can be represented by the conditional pmf:

$$
P_{Z_{1}^{n} \cdots Z_{M}^{n} Y_{1}^{n} \cdots Y_{N}^{n} \mid X_{1}^{n} X_{2}^{n}}=\prod_{i=1}^{n} P_{Z_{1, i} \cdots Z_{M, i} Y_{1, i} \cdots Y_{N, i} \mid X_{1, i} X_{2, i}} .
$$

An $\left(M_{1 n}, M_{2 n}, n\right)$-code for this channel consists of: two sets of messages, $\mathcal{M}_{1} \equiv\left\{1, \ldots, M_{1 n}\right\}$ and $\mathcal{M}_{2} \equiv\left\{1, \ldots, M_{2 n}\right\}$, two encoding functions, and $(N+M)$-decoding functions. The encoding function at source 1 (the primary source) assigns an n-sequence $x_{1}^{n}\left(W_{1}\right)$ to each message $W_{1}$ while the encoding function at source 2 assigns an $n$-sequence $x_{2}^{n}\left(W_{1}, W_{2}\right)$ to each pair of messages $\left(W_{1}, W_{2}\right) \in \mathcal{M}_{1} \times \mathcal{M}_{2}$. The secondary decoders indexed by $k \in[1: M]$ assign to each received sequence $Z_{k}^{n}$ an estimate message $\hat{W}_{2, k}$ while the primary decoders indexed by $j \in[1: N]$ each resort to a decoding function that assigns to each received sequence $Y_{j}^{n}$, an estimate message $\hat{W}_{1, j}$.

The probability of error is given by:

$$
P_{e}^{(n)} \equiv \mathbb{P}\left(\bigcup_{j \in[1: N]}\left\{\hat{W}_{1, j} \neq W_{1}\right\} \text { or } \bigcup_{k \in[1: M]}\left\{\hat{W}_{2, k} \neq W_{2}\right\}\right) .
$$

A rate pair $\left(R_{1}, R_{2}\right)$ is said to be achievable if there exists an $\left(M_{1 n}, M_{2 n}, n\right)$-code satisfying:

$$
\begin{aligned}
\liminf _{n \rightarrow \infty} & \frac{1}{n} \log _{2} M_{l n} \geq R_{l}, \quad l=\{1,2\}, \\
\limsup _{n \rightarrow \infty} P_{e}^{(n)} & =0 .
\end{aligned}
$$

The convex closure over all achievable rate pairs $\left(R_{1}, R_{2}\right)$ defines the capacity region.

Inner bound to the capacity region of the Multicast CIFC

In this section, we derive an inner bound to the capacity region of the CIFC, that generalizes Marton's inner bound for the $\mathrm{BC}$ in the presence of a helper, as shown in Fig. 1 . 
Theorem 1 (Inner bound). An inner bound to the capacity region of the CIFC consists in all rate pairs $\left(R_{1}, R_{2}\right)$ satisfying:

$$
\begin{aligned}
& * R_{1} \leq \min _{j \in[1: N]} I\left(Q_{1} X_{1} Q U ; Y_{j}\right) \\
& R_{2} \leq \min _{k \in[1: M]} I\left(Q V ; Z_{k} \mid Q_{1}\right)-I\left(Q V ; X_{1} \mid Q_{1}\right) \\
& R_{2} \leq \min _{j \in[1: N]} I\left(X_{1} U ; Y_{j} \mid Q_{1} Q\right)+\min _{k \in[1: M]} I\left(Q V ; Z_{k} \mid Q_{1}\right)-I\left(V ; X_{1} U \mid Q_{1} Q\right) \\
& R_{2} \leq \min _{j \in[1: N]} I\left(X_{1} Q U ; Y_{j} \mid Q_{1}\right)+\min _{k \in[1: M]} I\left(V ; Z_{k} \mid Q_{1} Q\right)-I\left(V ; X_{1} U \mid Q_{1} Q\right) \\
& R_{2} \leq \min _{j \in[1: N]} I\left(X_{1} Q U ; Y_{j} \mid Q_{1}\right)+\min _{k \in[1: M]} I\left(Q V ; Z_{k} \mid Q_{1}\right) \\
& -I\left(V ; X_{1} U \mid Q_{1} Q\right)-I\left(Q ; X_{1} \mid Q_{1}\right) \\
& R_{1}+R_{2} \leq \min _{j \in[1: N]} I\left(X_{1} U ; Y_{j} \mid Q_{1} Q\right)+\min _{k \in[1: M]} I\left(Q_{1} Q V ; Z_{k}\right)-I\left(V ; X_{1} U \mid Q_{1} Q\right) \\
& R_{1}+R_{2} \leq \min _{j \in[1: N]} I\left(Q_{1} X_{1} Q U ; Y_{j}\right)+\min _{k \in[1: M]} I\left(V ; Z_{k} \mid Q_{1} Q\right)-I\left(V ; X_{1} U \mid Q_{1} Q\right) \\
& R_{1}+R_{2} \leq \min _{j \in[1: N]} I\left(Q_{1} X_{1} Q U ; Y_{j}\right)+\min _{k \in[1: M]} I\left(Q V ; Z_{k} \mid Q_{1}\right) \\
& -I\left(V ; X_{1} U \mid Q_{1} Q\right)-I\left(Q ; X_{1} \mid Q_{1}\right) \\
& R_{1}+R_{2} \leq \min _{j \in[1: N]} I\left(X_{1} Q U ; Y_{j} \mid Q_{1}\right)+\min _{k \in[1: M]} I\left(Q_{1} Q V ; Z_{k}\right) \\
& -I\left(V ; X_{1} U \mid Q_{1} Q\right)-I\left(Q ; X_{1} \mid Q_{1}\right) \\
& R_{1}+2 R_{2} \leq \min _{j \in[1: N]} I\left(X_{1} Q U ; Y_{j} \mid Q_{1}\right)+\min _{k \in[1: M]} I\left(Q_{1} Q V ; Z_{k}\right) \\
& +\min _{k \in[1: M]} I\left(V ; Z_{k} \mid Q_{1} Q\right)-I\left(V ; X_{1} U \mid Q_{1} Q\right)-I\left(Q ; X_{1} \mid Q_{1}\right) \\
& R_{1}+2 R_{2} \leq \min _{j \in[1: N]} I\left(Q_{1} X_{1} Q U ; Y_{j}\right)+\min _{k \in[1: M]} I\left(Q V ; Z_{k} \mid Q_{1}\right) \\
& +\min _{k \in[1: M]} I\left(V ; Z_{k} \mid Q_{1} Q\right)-I\left(V ; X_{1} U \mid Q_{1} Q\right)-I\left(Q ; X_{1} \mid Q_{1}\right),
\end{aligned}
$$

for some joint $P D P_{Q_{1} X_{1} Q U V X_{2}}$ satisfying $\left(Q_{1} Q U V\right) \odot\left(X_{1}, X_{2}\right) \odot\left(Y_{1}, \cdots, Y_{N}, Z_{1}, \cdots, Z_{M}\right)$.

Remarks In the absence of the helper, i.e., when $X_{1}=Q_{1}=\emptyset$, the inner bound collapses to Marton's inner bound in the multicast setting with the common auxiliary $\mathrm{rv} Q$ and the two private ones $U$ and $V$. On the other hand, the variables $X_{1}$ and $Q_{1}$ account for rate-splitting at the primary source. The rate splitting at the secondary source is already contained in Marton's coding [16] with the common auxiliary $Q$. Thus, this inner bound combines both optimal coding schemes for the Broadcast Channel [16] and Interference Channel [17]. 
Proof: The proof is relegated to Appendix B.

In the sequel, we investigate two distinct classes of Multicast-CIFC. The Multi-Primary CIFC where we assume the existence of only one secondary receiver $M=1$, which will allow us to investigate the optimal interference mitigation techniques for primary receivers. Then, we investigate as well the dual class referred to as Multi-Secondary CIFC where we consider only one primary receiver $N=1$ and characterize the optimal interference mitigation technique within the set of secondary users.

\section{CAPACity Results For the Multi-Primary CIFC}

We refer to this class of Multicast CIFC as the Multi-Primary CIFC since it consists of only one secondary receiver, $Z$ as denoted in the sequel, and $N$ primary receivers as shown in Fig. 3 .

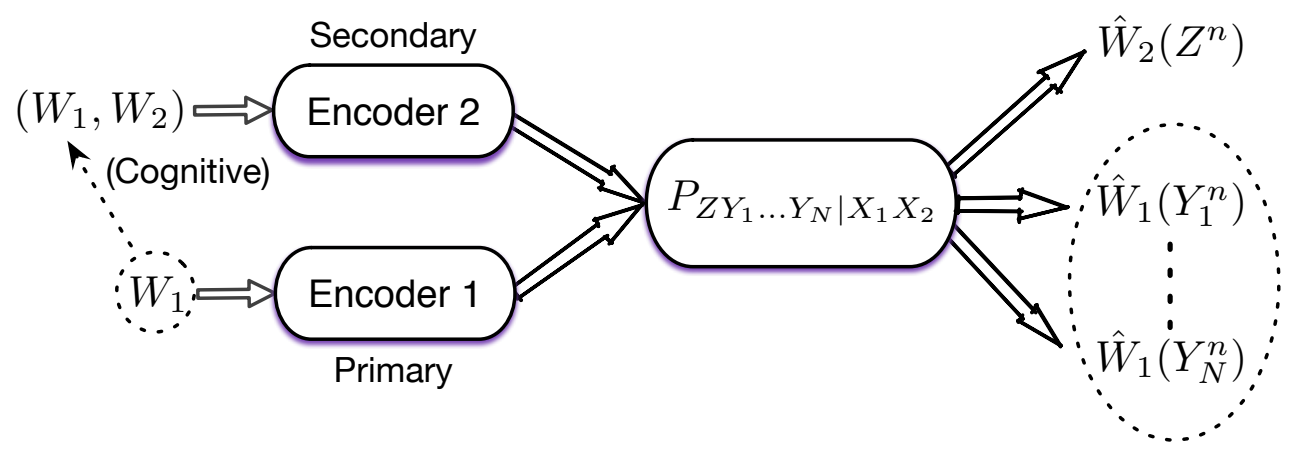

Fig. 3. The Multi-Primary Cognitive Interference Channel.

Our aim in this section is to characterize the capacity region of this class of channels under distinct interference regimes resorting to the inner bound stated in Theorem 1 and, in a specific regime, a more evolved decoding scheme -referred to as Interference Decoding- when (5) would be limited by the decoding behavior of the users.

\section{A. Capacity region of the Multi-Primary CIFC in the Very Strong Interference regime}

In this part of the work, we derive the capacity region of the Multi-Primary CIFC setting in the very strong interference regime. To this end, consider the multicast CIFC described in Fig 3.

Assumption 1 (Strong interference). The strong interference condition is defined as:

$$
\forall P_{X_{1} X_{2}}, \quad I\left(X_{2} ; Z \mid X_{1}\right) \leq \min _{j \in[1: N]} I\left(X_{2} ; Y_{j} \mid X_{1}\right) .
$$


Assumption 2 (Very strong interference). The very strong interference condition is defined as:

$$
\forall P_{X_{1} X_{2}}, \quad \min _{j \in[1: N]} I\left(X_{1} X_{2} ; Y_{j}\right) \leq I\left(X_{1} X_{2} ; Z\right)
$$

Theorem 2 (Very strong interference). The capacity region of the Multi-Primary CIFC satisfying assumptions (16) and (17) is given by the set of rate pairs $\left(R_{1}, R_{2}\right)$ that verify:

$$
\begin{aligned}
R_{2} & \leq I\left(X_{2} ; Z \mid X_{1}\right) \\
R_{1}+R_{2} & \leq \min _{j \in[1: N]} I\left(X_{1} X_{2} ; Y_{j}\right),
\end{aligned}
$$

for some $P D P_{X_{1} X_{2}}$.

Proof: The proof of achievability follows from rate region (5) letting $Q_{1}=X_{1}$ and $Q=$ $U=V=X_{2}$. This implies that all users $Y_{j}$ and $Z$ decode both signals $\left(X_{1}, X_{2}\right)$.

The obtained rate region is of the form:

$$
\begin{aligned}
* R_{1} & \leq \min _{j \in[1: N]} I\left(X_{1} X_{2} ; Y_{j}\right), \\
R_{2} & \leq I\left(X_{2} ; Z \mid X_{1}\right), \\
R_{2} & \leq \min _{j \in[1: N]} I\left(X_{2} ; Y_{j} \mid X_{1}\right), \\
R_{1}+R_{2} & \leq I\left(X_{1} X_{2} ; Z\right), \\
R_{1}+R_{2} & \leq \min _{j \in[1: N]} I\left(X_{1} X_{2} ; Y_{j}\right) .
\end{aligned}
$$

One can notice that 20 is redundant due to the sum-rate (24) while 22 is redundant due the strong interference assumption (16), and expression 23) is redundant by the very strong interference condition (17). As for the converse, it follows from the strong interference condition (16) alone and is given in Appendix C.

B. Capacity region of the Multi-Primary CIFC in the Very Weak Interference regime

We now give the capacity region of the under the very weak interference regime. To this end, se state the next assumption.

Assumption 3 (Weak interference). Let $\mathcal{P}_{w}$ be the set of all joint PDs $P_{U X_{1} X_{2}}$ which satisfy the following Markov chain:

$$
U \odot\left(X_{1}, X_{2}\right) \multimap\left(Y_{1}, \cdots, Y_{N}, Z_{1}, \cdots, Z_{M}\right)
$$


The weak interference regime is defined as:

$$
\forall j \in[1: N], \quad \forall P_{U X_{1} X_{2}} \in \mathcal{P}_{w}, \quad I\left(U ; Y_{j} \mid X_{1}\right) \leq I\left(U ; Z \mid X_{1}\right)
$$

Assumption 4 (Very weak interference). The very weak interference regime is to verify (26) while to further satisfy:

$$
\forall j \in[1: N], \quad \forall P_{U X_{1} X_{2}} \in \mathcal{P}_{w}, \quad I\left(U X_{1} ; Y_{j}\right) \leq I\left(U X_{1} ; Z\right)
$$

The intuitive extension of the result of the standard CIFC (non-multicast CIFC with $N=1$ ) to an arbitrary number of users $N$ will prove to be optimal for this regime as well. Yet, the proof of the resulting capacity region presents a major difficulty in the converse part. Indeed, the multicast nature of the transmission prevents from resorting to Csiszár \& Körner's identity that was originally used in [4], [3]. To this end, an alternative proof of converse is required herein.

Theorem 3 (Very weak interference). The capacity region of the Multi-primary CIFC in very weak interference regime is given by the set of rate pairs satisfying:

$$
\begin{aligned}
& R_{1} \leq \min _{j \in[1: N]} I\left(X_{1} U ; Y_{j}\right) \\
& R_{2} \leq I\left(X_{2} ; Z \mid X_{1} U\right)
\end{aligned}
$$

for some arbitrarily $P D P_{U X_{1} X_{2}}$ satisfying $U \odot\left(X_{1}, X_{2}\right) \multimap\left(Y_{1}, \cdots, Y_{N}, Z\right)$.

Proof: The achievability follows from the inner bound in (5) by letting $Q=U, Q_{1}=$ $\left(X_{1}, U\right)$, and $V=X_{2}$ which amounts to enabling decoders $Y_{j}$ to decode only their useful signal while user $Z$ decodes both signals $\left(X_{1}, X_{2}\right)$. We end up with the inner bound:

$$
\begin{aligned}
R_{1} & \leq \min _{j \in[1: N]} I\left(X_{1} U ; Y_{j}\right), \\
R_{2} & \leq I\left(X_{2} ; Z \mid X_{1} U\right), \\
R_{1}+R_{2} & \leq I\left(X_{1} X_{2} ; Z\right) .
\end{aligned}
$$

Using the very weak interference condition (27), the sum-rate bound is redundant. Hence, the achievability is proved. As for the converse, the proof is more evolved and is presented in Appendix $\mathrm{D}$. The proof is valid under the loose condition of weak interference assumption alone (26) and originates from noticing that this condition implies a "conditional less-noisiness" [18] order between $Y_{j}$ and $Z$ conditioned on $X_{1}$. 
In both previously investigated regimes of very weak and very strong interference, the inner bound (5) proves to be optimal and an extension of the intuitive coding schemes to an arbitrary number of users turns out to be optimal, though it might have required to write a new outer bound to prove the optimality. In the sequel, we investigate a setting that did not arise in the non-multicast CIFC where $N=1$ and that will turn out to require a more evolved coding scheme that can be encompassed with the inner bound (5).

C. Capacity region of the Multi-Primary CIFC in the Mixed Very Weak/Strong Interference regime

We consider a Multi-Primary CIFC where we can partition the multicast set of users $Y_{j}$, $j \in[1: N]$, into two subsets. A subset $\mathcal{W}$ where all users are in weak interference, and a subset $\mathcal{S}$ where users are in the strong interference regime, however, very weak or very strong interference can be verified in either of the sets, without being imposed on both sets.

Assumption 5 (Mixed very weak/strong interference). The mixed very weak/strong interference regime is defined by the following conditions:

$$
\begin{aligned}
\forall j \in \mathcal{W} \quad, \quad I\left(U ; Y_{j} \mid X_{1}\right) \leq I\left(U ; Z \mid X_{1}\right), \\
\forall j \in \mathcal{S} \quad, \quad I\left(X_{2} ; Z \mid X_{1}\right) \leq I\left(X_{2} ; Y_{j} \mid X_{1}\right), \\
\min _{j \in \mathcal{S}} I\left(X_{1} X_{2} ; Y_{j}\right) \leq I\left(X_{1} X_{2} ; Z\right) \text { or } \min _{j \in \mathcal{W}} I\left(U X_{1} ; Y_{j}\right) \leq I\left(U X_{1} ; Z\right),
\end{aligned}
$$

for all $P_{U X_{1} X_{2}}$ that satisfied the Markov chain:

$$
U \odot\left(X_{1}, X_{2}\right) \multimap\left(Y_{1}, \cdots, Y_{N}, Z_{1}, \cdots, Z_{M}\right) .
$$

Before enunciating the main result of this section, the following remark is crucial. The set of users $Y_{j}$ that experience very strong interference would require decoding the interfering codeword $X_{2}$, whilst those that experience very weak interference are required to decode only their intended useful codewords $U$ and $X_{1}$. User $Z$ is required to decode all codewords in both interference regimes. As it is enunciated, the inner bound (5) fails in encompassing this differentiated behaviour of users $Y_{j}$ since it relies on a similar decoding strategy for all users $Y_{j}$. Thus, the need for a more evolved decoding strategy arises, which justifies the introduction of the idea of "Interference Decoding". 
This decoding strategy was first investigated by the authors for a Compound Broadcast Channel [13] which can be regarded as a multicast setting where the many users of the multicast are designated by the many possible channel instances of the Broadcast setting. Interference Decoding allows each user in the multicast group $Y_{j}$ to decode/ or not the interfering codeword $X_{2}$, and thus, provides for a differentiated decoding at all users. Resorting to this idea allows to state the following result.

Theorem 4 (Mixed very weak/strong interference). The capacity region of the Multi-primary CIFC satisfying conditions (33)-35), is given by the set of rate pairs satisfying:

$$
\begin{aligned}
R_{1} & \leq \min _{j \in \mathcal{W}} I\left(U X_{1} ; Y_{j}\right), \\
R_{2} & \leq I\left(X_{2} ; Z \mid U X_{1}\right), \\
R_{1}+R_{2} & \leq \min _{j \in \mathcal{S}} I\left(X_{1} X_{2} ; Y_{j}\right),
\end{aligned}
$$

for some joint input $P D P_{U X_{1} X_{2}}$ such that $U \odot\left(X_{1}, X_{2}\right) \multimap\left(Y_{1}, \cdots, Y_{N}, Z\right)$.

Proof: The converse proof follows in the exact same manner as the converse proof of both weak and strong interference cases. As for the achievability part, it is more involved and requires introducing the idea of Interference Decoding. The decoders $Y_{j}$ with $j \in \mathcal{W}$ will choose to decode only the useful signal $U$ and $X_{1}$ while the users that are in strong interference, i.e $Y_{j}$ with $j \in \mathcal{S}$, will decode all signals transmitted by source 2 and source 1 , that is: $\left(U, X_{1}, X_{2}\right)$. User $Z$ is allowed to decode all codewords as well. Proof details can be found in Appendix E.

\section{Capacity results for the Gaussian Multi-Primary CIFC}

Consider the following Gaussian Multicast CIFC model as shown in Fig 4

$$
\begin{aligned}
\forall j \in[1: N], \quad Y_{j} & =b_{j} X_{2}+X_{1}+n_{j}, \\
Z & =X_{2}+a X_{1}+n_{z},
\end{aligned}
$$

where $b_{j}, j \in[1: N]$, and $a$ are real numbers, and where $\left(n_{1}, \cdots, n_{N}\right)$, and $n_{z}$ are additive white Gaussian noise components with powers $N_{1}=\cdots=N_{N}=N_{z}=1$.

In this section, we derive the capacity region for several different regimes of the Gaussian Multi-Primary CIFC. 


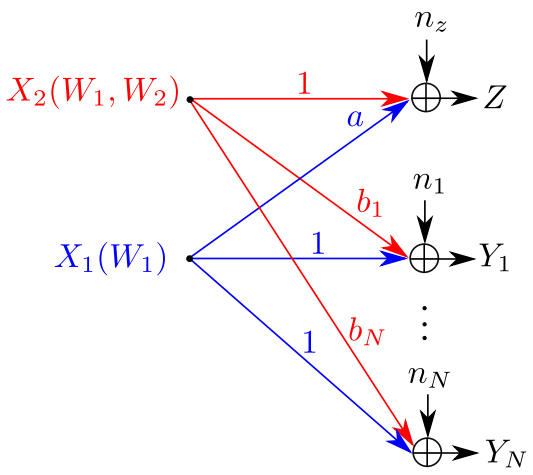

Fig. 4. The Gaussian Multi-Primary Cognitive Interference Channel.

Theorem 5 (Gaussian Multi-Primary CIFC). The capacity region of the Gaussian Multi-Primary CIFC is characterized in the following regimes:

1) The capacity region of the very strong interference regime is given by the set of rate pairs that satisfy:

$$
\begin{aligned}
& \forall j \in[1: N],\left|b_{j}\right| \geq 1, \\
& \forall \rho \in[-1: 1], \min _{j \in[1: N]}\left\{\left(1-a^{2}\right) P_{1}+\left(b_{j}^{2}-1\right) P_{2}+2 \rho\left(b_{j}-a\right) \sqrt{P_{1} P_{2}}\right\} \leq 0,
\end{aligned}
$$

consists in the set of rate pairs satisfying:

$$
\begin{aligned}
R_{2} & \leq \frac{1}{2} \log _{2}\left(1+\left(1-\rho^{2}\right) P_{2}\right), \\
R_{1}+R_{2} & \leq \frac{1}{2} \min _{j \in[1: N]} \log _{2}\left(1+b_{j}^{2} P_{2}+P_{1}+2 b_{j} \rho \sqrt{P_{1} P_{2}}\right),
\end{aligned}
$$

for some $\rho \in[-1: 1]$.

2) The capacity region of the weak interference regime is given by the set of rate pairs that satisfy:

$$
\forall j \in[1: N],\left|b_{j}\right| \leq 1,
$$

is given by the set of rate pairs satisfying:

$$
\begin{aligned}
& R_{1} \leq \frac{1}{2} \max _{\rho \in[-1: 1]} \min _{j \in[1: N]} \log _{2}\left(\frac{1+b_{j}^{2} P_{2}+P_{1}+2 b_{j} \rho \sqrt{(1-\eta) P_{1} P_{2}}}{1+b_{j}^{2} \eta P_{2}}\right), \\
& R_{2} \leq \frac{1}{2} \log _{2}\left(1+\eta P_{2}\right),
\end{aligned}
$$

for some $\eta \in[0: 1]$. 
3) The capacity region of the mixed weak/very strong interference regime, where $[1: N]=$ $\mathcal{S} \cup \mathcal{W}$ and

$$
\begin{aligned}
\forall j \in \mathcal{W},\left|b_{j}\right| \leq 1, \\
\forall j \in \mathcal{S},\left|b_{j}\right| \geq 1, \\
\forall \rho \in[-1: 1], \min _{j \in \mathcal{S}}\left\{\left(1-a^{2}\right) P_{1}+\left(b_{j}^{2}-1\right) P_{2}+2 \rho\left(b_{j}-a\right) \sqrt{P_{1} P_{2}}\right\} \leq 0,
\end{aligned}
$$

is given by the set of rate pairs that satisfy:

$$
\begin{aligned}
R_{1} & \leq \frac{1}{2} \min _{j \in \mathcal{W}} \log _{2}\left(\frac{1+b_{j}^{2} P_{2}+P_{1}+2 b_{j} \rho \sqrt{(1-\eta) P_{1} P_{2}}}{1+b_{j}^{2} \eta P_{2}}\right), \\
R_{2} & \leq \frac{1}{2} \log _{2}\left(1+\eta P_{2}\right), \\
R_{1}+R_{2} & \leq \frac{1}{2} \min _{j \in \mathcal{S}} \log _{2}\left(1+b_{j}^{2} P_{2}+P_{1}+2 b_{j} \rho \sqrt{(1-\eta) P_{1} P_{2}}\right),
\end{aligned}
$$

for some $\eta \in[0: 1]$ and $\rho \in[-1: 1]$.

Proof: A detailed proof of this theorem is given in Appendix F However, hereafter the outline of proof for every interference regime.

1) Very strong interference regime. The achievability scheme consists in letting the two Gaussian codewords $\left(X_{1}, X_{2}\right)$ to be arbitrarily correlated, defining thus: $\mathbb{E}\left[X_{1} X_{2}\right]=\rho \sqrt{P_{1} P_{2}}$. The proof of converse can be deduced from Rini et al.'s work [9] by showing that their suggested outer bound can be generalized to an arbitrary number of primary users.

2) Weak interference regime. Note here first that we no longer consider very weak interference but we rather investigate the looser condition of weak interference. The reason being that, in the Gaussian case, we can resort to Dirty-Paper Coding to eliminate the interference $\left(U, X_{1}\right)$ at decoder $Z$, alleviating thus the decoding constraint at user $Z$, i.e.,

$$
I\left(U X_{1} ; Y_{j}\right) \leq I\left(U X_{1} ; Z\right) .
$$

The achievability part is briefly described as follows: Split the power $P_{2}$ into two parts $\eta P_{2}$ and $(1-\eta) P_{2}$. Generate a Gaussian ARV $U$ with variance $(1-\eta) P_{2}$ and let it arbitrarily correlated to $X_{1}$ through the correlation coefficient $\rho$. Generate a Gaussian ARV $V$ that dirty paper codes jointly the interference component $X_{u}+a X_{1}$. The converse part follows from Entropy Power Inequality along with some analytic manipulations. 
3) Mixed weak/very strong interference regime. The achievability relies on the idea of Interference Decoding along with a strategic combination of both coding schemes for weak and very strong interference regimes. As for the converse, it follows similarly from combining entropy power inequality and entropy maximization techniques.

In the sequel we give a simplified expression of the capacity regions enunciated above for some setting where the interference components seen at the primary decoders are coherent, i.e. all $b_{j}$ 's are of the same sign, as opposed to the non-coherent interference case where $b_{j}$ 's might be of distinct signs.

Corollary 1. 1) Provided that all gains $b_{j}$ 's share the same sign, i.e., coherent weak interference, the capacity region in weak interference regime is given by the set of rate pairs satisfying:

$$
\mathcal{C}_{W I}(\eta):\left\{\begin{aligned}
R_{1} & \leq \frac{1}{2} \min _{j \in[1: N]} \log _{2}\left(\frac{1+b_{j}^{2} P_{2}+P_{1}+2\left|b_{j}\right| \sqrt{(1-\eta) P_{1} P_{2}}}{1+b_{j}^{2} \eta P_{2}}\right), \\
R_{2} & \leq \frac{1}{2} \log _{2}\left(1+\eta P_{2}\right) .
\end{aligned}\right.
$$

Moreover, the capacity region consists of the intersection of all capacity regions of the CIFCs $\left(Z, Y_{j}\right)$.

2) Provided that all gains $b_{j}$ 's share the same sign, i.e., coherent strong interference, the capacity region is given by the set of rate pairs satisfying:

$$
\mathcal{C}_{S I}(\eta):\left\{\begin{aligned}
R_{2} & \leq \frac{1}{2} \log _{2}\left(1+\eta P_{2}\right) \\
R_{1}+R_{2} & \leq \frac{1}{2} \log _{2}\left(1+b_{*}^{2} P_{2}+P_{1}+2\left|b_{*}\right| \sqrt{P_{1} P_{2}}\right)
\end{aligned}\right.
$$

where

$$
\left|b_{*}\right| \equiv \min _{j \in[1: N]}\left|b_{j}\right| .
$$

Moreover, the capacity region consists in the intersection of all capacity regions of the CIFCs $\left(Z, Y_{j}\right)$.

3 ) Provided that all gains $b_{j}$ 's share the same sign, i.e., coherent mixed interference, the capacity region is given by the set of rate pairs satisfying:

$$
\mathcal{C}_{M I}(\eta):\left\{\begin{aligned}
R_{1} & \leq \frac{1}{2} \min _{j \in \mathcal{W}} \log _{2}\left(\frac{1+b_{j}^{2} P_{2}+P_{1}+2\left|b_{j}\right| \sqrt{(1-\eta) P_{1} P_{2}}}{1+b_{j}^{2} \eta P_{2}}\right), \\
R_{2} & \leq \frac{1}{2} \log _{2}\left(1+\eta P_{2}\right), \\
R_{1}+R_{2} & \leq \frac{1}{2} \min _{j \in \mathcal{S}} \log _{2}\left(1+b_{j}^{2} P_{2}+P_{1}+2\left|b_{j}\right| \sqrt{(1-\eta) P_{1} P_{2}}\right) .
\end{aligned}\right.
$$




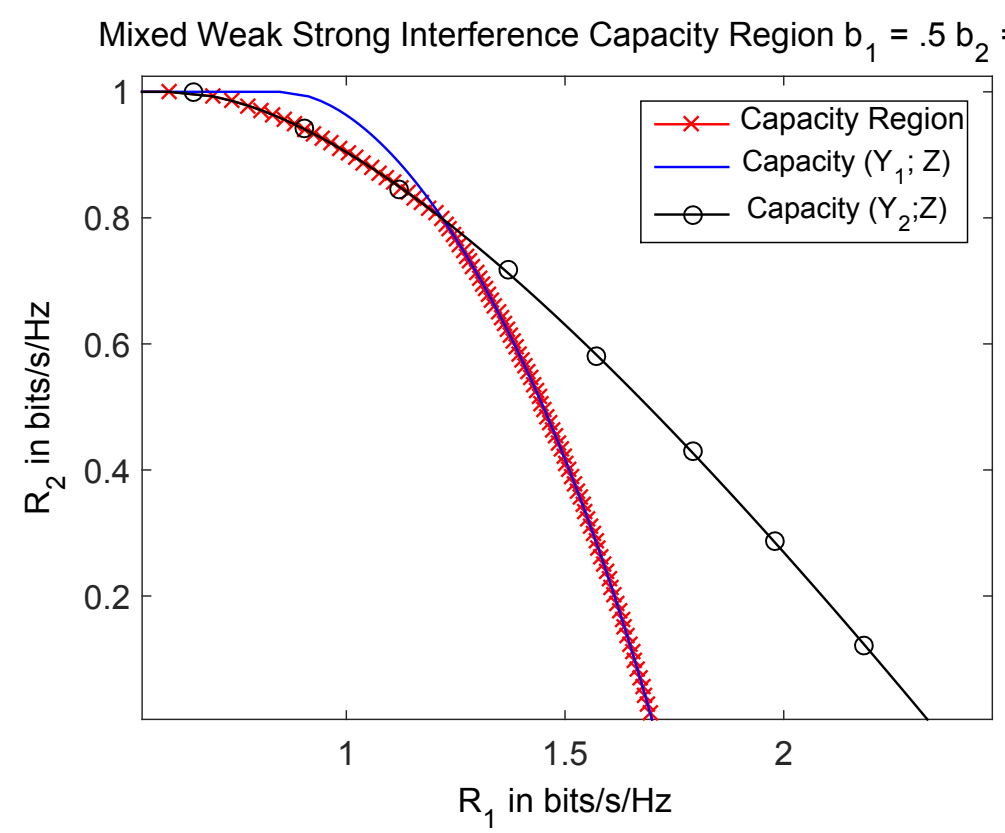

Fig. 5. The Coherent Multi-Primary Cognitive Interference Channel.

In addition, the capacity region is the intersection of the single capacity regions.

Proof: The proof of this corollary is given in Appendix F.

To illustrate the results of this corollary, we plot the capacity region for two settings, in both coherent and non-coherent interference regimes as shown in Fig. 5 and Fig. 6

In this section, we have investigated the Multi-Primary CIFC which consists of one secondary user $Z$ and $N$ primary users $Y_{j}, j \in[1: N]$. The aim was to characterize the capacity region in different interference regimes and infer on the optimal required interference mitigation techniques. The need for a more evolved decoding technique arises in the mixed interference case, as opposed to the very weak and very strong interference regimes where the intuitive extension of the optimal non-multicast scheme turned out to be optimal for the multicast setting as well. In the sequel, we investigate the Multi-Secondary CIFC and characterize in a similar manner the optimal interference mitigation techniques in distinct interference regimes.

\section{CAPACity Results For the Multi-Secondary CIFC}

In this section, we investigate the dual class of Multi-Primary CIFC referred to as the MultiSecondary CIFC, as depicted in Fig. 7. In such a setting, we assume the existence of only 


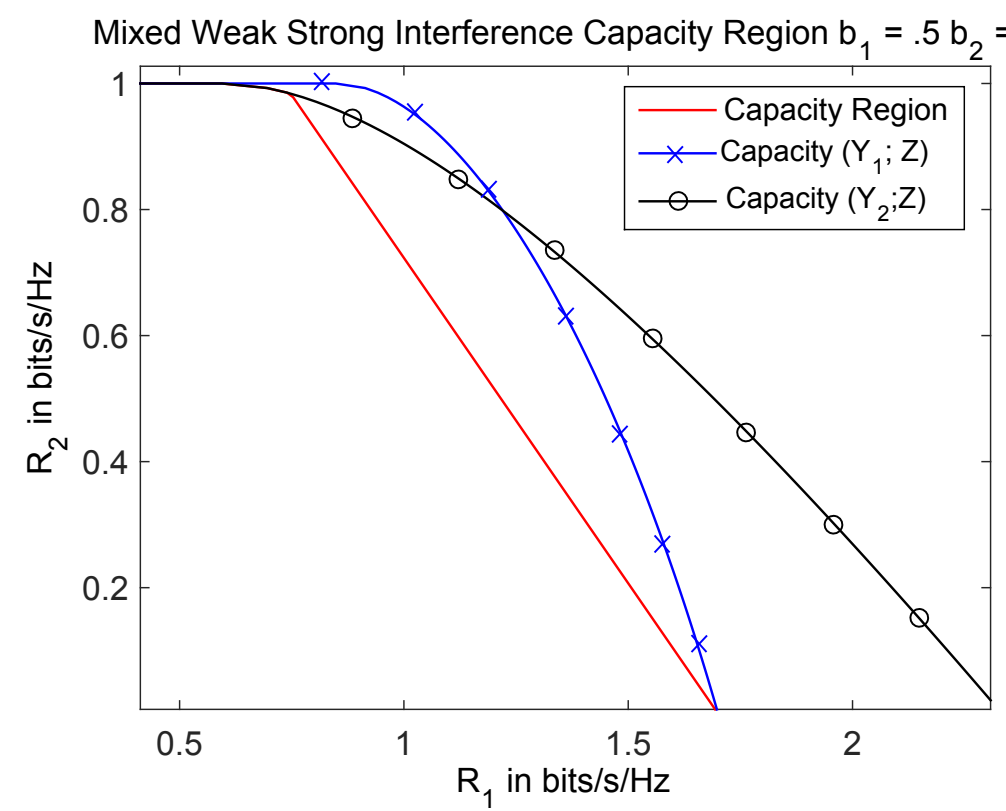

Fig. 6. The Non-Coherent Multi-Primary Cognitive Interference Channel.

one primary receiver but as many as $M$ secondary decoders $Z_{k}$ with $k \in[1: M]$. Similar

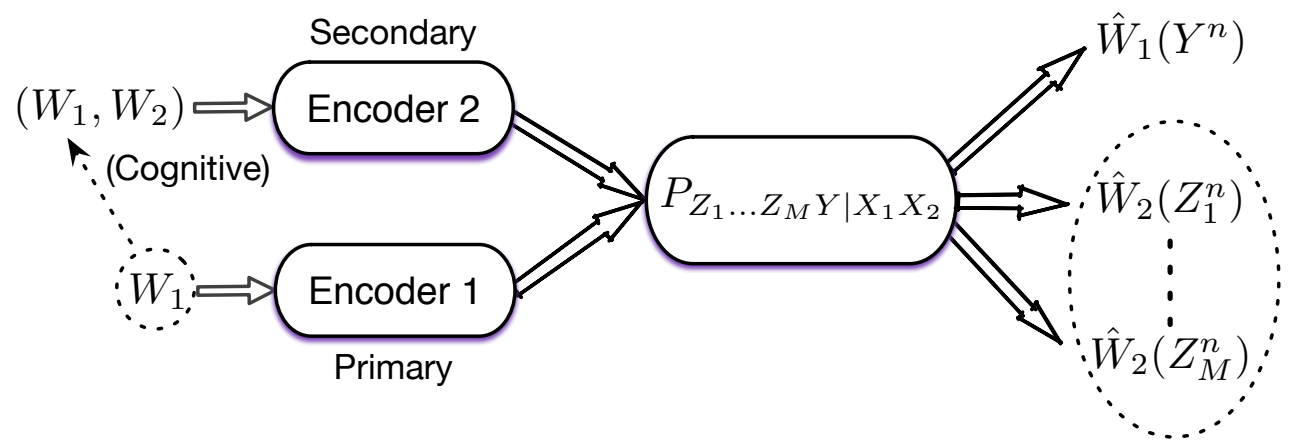

Fig. 7. The Multi-Secondary Cognitive Interference Channel.

to the Multi-Primary CIFC, our aim is to characterize the capacity region for several different interference regimes which will be defined in a somewhat dual manner to the previous section. It is worth mentioning at this point that, due to the asymmetry of the roles played by both primary and secondary encoders/receivers, the results of the two sections, though they might exhibit some similarities, originate from fundamentally different challenges to solve as will be later elaborated on. 
A. Capacity region of the Multi-Secondary CIFC in the Very Strong Interference regime Assumption 6 (Very strong interference). The very strong interference regime is defined by:

$$
\begin{array}{r}
(\forall k \in[1: M]), I\left(X_{2} ; Z_{k} \mid X_{1}\right) \leq I\left(X_{2} ; Y \mid X_{1}\right), \\
I\left(X_{1} X_{2} ; Y\right) \leq I\left(X_{1} X_{2} ; Z_{k}\right) .
\end{array}
$$

for all joint PDs $P_{X_{1} X_{2}}$.

It is worth to mention the above assumption implies that all users $Z_{k}$ and $Y$ are assumed to experience strong interference, and thus can all of them decode the interference.

Theorem 6 (Very strong interference). The capacity region of the Multi-Secondary CIFC in very strong interference is defined by all rate pairs verifying:

$$
\begin{aligned}
R_{2} & \leq \min _{k \in[1: M]} I\left(X_{2} ; Z_{k} \mid X_{1}\right), \\
R_{1}+R_{2} & \leq I\left(X_{1} X_{2} ; Y\right),
\end{aligned}
$$

for some joint $P D P_{X_{1} X_{2}}$.

Proof: The inner bound follows from superposing the codeword letting similarly to previously $Q_{1}=X_{1}, V_{1}=V_{2}=V$ and $Q=U=V=X_{2}$, i.e., allowing all users to decode all codewords. The proof of converse is standard and follows along similar lines to the proof of Multi-Primary CIFC presented in Appendix C.

B. Capacity region of the Multi-Secondary CIFC in the Very Weak Interference Regime Assumption 7 (Very weak interference). The very weak interference regime is defined by

$$
\begin{aligned}
& I\left(U ; Y \mid X_{1}\right) \leq \min _{k \in[1: M]} I\left(U ; Z_{k} \mid X_{1}\right), \\
& I\left(U X_{1} ; Y\right) \leq \min _{k \in[1: M]} I\left(U X_{1} ; Z_{k}\right),
\end{aligned}
$$

for all $P_{U X_{1} X_{2}}$ verifying $U-\left(X_{1}, X_{2}\right) \odot\left(Y, Z_{1}, \ldots, Z_{M}\right)$.

Theorem 7 (Very weak interference). The capacity region of the Multi-Secondary CIFC in very weak interference regime is defined by all rate pairs verifying:

$$
R_{1} \leq I\left(U X_{1} ; Y\right)
$$




$$
R_{2} \leq \min _{k \in[1: M]} I\left(X_{2} ; Z_{k} \mid U X_{1}\right),
$$

where $U \odot\left(X_{1}, X_{2}\right) \multimap\left(Y, Z_{1}, \ldots, Z_{M}\right)$.

Proof: The inner bound follows from letting $Q_{1}=\left(U, X_{1}\right), V_{1}=V_{2}=V=X_{2}$ and using both constraints (64) and 65). The converse proof is more challenging, is dual to the proof made for the Multi-Primary CIFC and is presented in Appendix $\mathrm{G}$.

C. Capacity region of the Multi-Secondary CIFC in the Mixed Very Weak/Strong Interference Regime

Assumption 8 (Mixed very weak/strong interference). Assume the set of secondary users can be partitioned in two subsets: $\mathcal{S}$ corresponding to the set of users experiencing very strong interference, and $\mathcal{W}$ indicating the set of users that experience very weak interference. Accordingly, the mixed very weak/strong interference regime is defined by the set of inequalities:

$$
\begin{aligned}
I\left(U ; Y \mid X_{1}\right) & \leq \min _{k \in \mathcal{W}} I\left(U ; Z_{k} \mid X_{1}\right), \\
I\left(U X_{1} ; Y\right) & \leq \min _{k \in \mathcal{W}} I\left(U X_{1} ; Z_{k}\right), \\
(\forall j \in \mathcal{S}), I\left(X_{2} ; Z_{k} \mid X_{1}\right) & \leq I\left(X_{2} ; Y \mid X_{1}\right), \\
I\left(X_{1} X_{2} ; Y\right) & \leq \min _{k \in \mathcal{S}} I\left(X_{1} X_{2} ; Z_{k}\right),
\end{aligned}
$$

for all joint $P D S P U X_{1} X_{2}$ such that $U-\left(X_{1}, X_{2}\right) \multimap\left(Y, Z_{1}, \ldots, Z_{M}\right)$.

Theorem 8 (Mixed very weak/strong interference). The capacity region of the Multi-Secondary CIFC in mixed very weak/ strong interference regime is the set of rate pairs satisfying:

$$
\begin{aligned}
R_{2} & \leq \min _{k \in \mathcal{S}} I\left(X_{2} ; Z_{k} \mid X_{1}\right), \\
R_{1}+R_{2} & \leq I\left(X_{1} X_{2} ; Y\right),
\end{aligned}
$$

for some joint $P D P_{X_{1} X_{2}}$.

Proof: Converse proof is straightforward. Achievability follows from evaluating the rate region $\mathcal{M}$ with the choice $V_{1}=V_{2}=\emptyset, Q_{1}=X_{1}$, and $Q=U=V=X_{2}$ which is the optimal scheme for very strong interference. Then, we note that:

$$
\text { Expressions (68) and 70] } \Rightarrow \min _{k \in \mathcal{S}} I\left(X_{2} ; Z_{k} \mid X_{1}\right) \leq \min _{k \in \mathcal{W}} I\left(X_{2} ; Z_{k} \mid X_{1}\right) \text {, }
$$




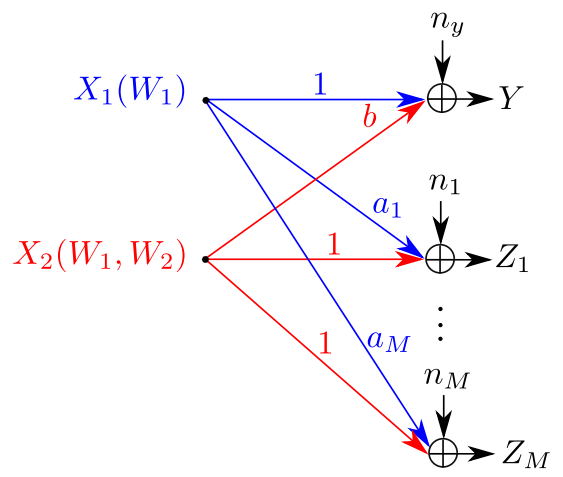

Fig. 8. The Gaussian Multi-Secondary Cognitive Interference Channel.

$$
\text { Expressions (69) and (71) } \Rightarrow I\left(X_{1} X_{2} ; Y\right) \leq \min _{k \in \mathcal{S} \cup \mathcal{W}} I\left(X_{1} X_{2} ; Z_{k}\right) \text {, }
$$

which leads to the capacity region formulation.

Unlike the Multi-primary CIFC, the inner bound (5) is optimal for all discrete memoryless interference regimes for which capacity region was characterized. Yet, the Gaussian counterpart, i.e., the Gaussian Multi-Secondary CIFC, appears to be more challenging, especially in the weak interference regime. Hence, it will require the introduction of a more evolved encoding technique.

D. Capacity results for the Gaussian Multi-Secondary CIFC

Let us consider the following channel model as depicted in Fig. 8;

$$
\begin{aligned}
& Y=X_{1}+b X_{2}+N, \\
& Z_{1}=X_{2}+a_{1} X_{1}+N_{1}, \\
& Z_{2}=X_{2}+a_{2} X_{1}+N_{2},
\end{aligned}
$$

where $\left(N, N_{1}, N_{2}\right)$ are independent and $N, N_{1}, N_{2} \sim \mathcal{N}(0,1)$.

1) The Very Strong Interference regime:

Assumption 9 (Very strong interference). The very strong interference regime in the Gaussian setting is defined by the couple of constraints:

$$
\begin{aligned}
& |b|>1, \forall \rho \in[-1: 1] \quad, \forall k \in[1: 2], \\
& \left(1-a_{k}^{2}\right) P_{1}+\left(b^{2}-1\right) P_{2}+2 \rho\left(b-a_{k}\right) \sqrt{P_{1} P_{2}} \leq 0 .
\end{aligned}
$$


The capacity region in this case is given by the following.

Corollary 2 (Very strong interference). The capacity of the Gaussian Multicast CIFC in the very strong interference regime is given by the set of rate pairs satisfying:

$$
\begin{aligned}
R_{2} & \leq \frac{1}{2} \log _{2}\left(1+\eta P_{2}\right) \\
R_{1}+R_{2} & \leq \frac{1}{2} \log _{2}\left(1+b^{2} P_{2}+P_{1}+2|b| \sqrt{(1-\eta) P_{1} P_{2}}\right)
\end{aligned}
$$

for some $\eta \in[0: 1]$.

Proof: The inner bound follows from noting that, since conditioned on $X_{1}$ all channels $Z_{j}$ have equal statistics, the index $j$ does not play a role in the capacity region. The proof follows along similar lines as in [9] since it is equivalent to a non-mutlticast CIFC.

2) The Weak Interference regime:

Assumption 10 (Weak Interference regime). The weak interference regime is defined by $|b| \leq 1$ which is equivalent to satisfy expression (64).

Note here, that we again investigate for the Gaussian case, a weak interference regime as opposed to the discrete memoryless case where we can only characterize the capacity region of the very weak interference regime.

Indeed, in the non-multicast setting, $Z_{1}=Z_{2}=Z$, i.e., $a_{1}=a_{2}=a$, the optimal strategy is to apply Dirty-Paper Coding by letting the codeword destined to user $Z$, i.e. $V$, precode against interference $\left(X_{u}, X_{1}\right)$ which is the signal intended to user $Y$. It is only at this condition that we can alleviate the conditions of very weak interference to that of weak interference (64) since the sum-rate decoding constraint is no longer necessary. However, in the multicast setting, by setting $X_{2}=X_{v}+X_{u}$, we have that:

$$
Z_{k}=X_{v}+\overbrace{X_{u}}^{\text {Common IFC }}+\overbrace{a_{k} X_{1}}^{\text {Private IFC }}+N_{k},
$$

the transmission to both users $Z_{k}$ experiences different interference components $X_{u}+a_{1} X_{1}$ and $X_{u}+a_{2} X_{1}$, it thus becomes inefficient to apply only a "common" DPC scheme.

We remark that a similar challenging setting, though less general, was investigated in [14] when dealing with simultaneous transmissions to a single-user having uncertainty about two different 
interferences, e.g. $a_{1} S^{n}$ and $a_{2} S^{n}$, and where tight upper and lower bounds on the capacity region of such a setting are derived, but under the assumption that the sequence $S^{n}$ is i.i.d and Gaussian distributed. Besides having to deal with similar uncertainty about the interfering sequence, we cannot assume an i.i.d. distribution for the input $X_{1}$, which renders the analysis of the inner and the outer bounds even more challenging.

The authors resort here to previous work presented as "Multiple Description Coding" that was investigated within the framework of the Compound Broadcast Channel [13] but that can be equivalently stated in the Multicast setting as well. In this precoding scheme, we introduce two private descriptions, each decoded by a distinct destination $Z_{k}$, leading thus to the rate region $\mathcal{R}$ defined by:

$$
\begin{aligned}
R_{2} & \leq \min _{k \in[1: 2]}\left\{I\left(V V_{k} ; Z_{k}\right)-I\left(V V_{k} ; U X_{1}\right)\right\} \\
2 & R_{2} \leq \sum_{k \in[1: 2]}\left[I\left(V V_{k} ; Z_{j}\right)-I\left(V V_{k} ; U X_{1}\right)\right]-I\left(V_{1} ; V_{2} \mid V U X_{1}\right) \\
R_{1} & \leq I\left(U X_{1} ; Y\right) .
\end{aligned}
$$

Our aim is to show that, compared to the inner bound based on "Common Description" coding:

$$
\begin{aligned}
& R_{2} \leq \min _{k \in[1: 2]} I\left(V ; Z_{k}\right)-I\left(V ; U X_{1}\right), \\
& R_{1} \leq I\left(U X_{1} ; Y\right),
\end{aligned}
$$

multiple description coding can strictly enhance the rates at users $Z_{k}$. To this end, we construct two DPC schemes based on the two different ideas: MD-DPC and CD-DPC, and compare their respective performances. In the sequel, let:

$$
\left(X_{1}, X_{u}\right) \sim \mathcal{N}\left(\mathbf{0}, \Sigma_{X_{1} X_{u}}\right) \quad \text { with } \quad \Sigma_{X_{1} X_{u}}=\left(\begin{array}{cc}
P_{1} & \rho \sqrt{P_{1} P_{u}} \\
\rho \sqrt{P_{1} P_{u}} & P_{u}
\end{array}\right)
$$

so that $P_{u}=(1-\eta) P_{2}$ and let $P_{v}=\eta P_{2}$. Thus,

$$
R_{1} \leq I\left(U X_{1} ; Y\right)=\frac{1}{2} \log _{2}\left(\frac{b^{2} P_{2}+P_{1}+2 b \rho \sqrt{P_{1}(1-\eta) P_{2}}+1}{b^{2} \eta P_{2}+1}\right) .
$$

3) Common Description Dirty-Paper Coding: Consider the CIFC without multicasting, and set the auxiliary random variable:

$$
V=X_{v}+\gamma X_{u}+\alpha X_{1} .
$$


The maximal rate $R_{2}$ achieved by this DPC scheme is then:

$$
\begin{aligned}
\max _{\gamma, \alpha}\left[I(V ; Z)-I\left(V ; X_{u} X_{1}\right)\right] & =I\left(X_{v} ; Z \mid X_{u} X_{1}\right) \\
& =\frac{1}{2} \log _{2}\left(P_{v}+1\right),
\end{aligned}
$$

and is achieved with: $\gamma_{o p t}=\frac{P_{v}}{P_{v}+1}$ and $\alpha_{o p t}=a \frac{P_{v}}{P_{v}+1}$. However, in presence of a multicast setting, $\gamma$ cannot be chosen optimal for both channels, which justifies the appellation of common interference. For instance, the private interference cannot be suppressed entirely for both channels at once since it would require that $\alpha_{o p t, 1}=a_{1} \frac{P_{v}}{P_{v}+1}$ and $\alpha_{o p t, 2}=a_{2} \frac{P_{v}}{P_{v}+1}$.

Corollary 3 (CD-DPC lower bound). The maximal rate $R_{2}$ achieved under the CD-DPC scheme (88) is given by:

$$
\begin{aligned}
\max _{\alpha, \gamma} \min _{k \in[1: 2]}\left[I\left(V ; Z_{k}\right)\right. & \left.-I\left(V ; U X_{1}\right)\right]=\frac{1}{2} \log _{2}\left(P_{v}+\right) \\
& -\frac{1}{2} \log _{2}\left(\frac{P_{1} P_{v}\left(P_{v}+\left(1-\rho^{2}\right) P_{u}+1\right)\left(a_{1}-a_{2}\right)^{2}}{\left(P_{v}+1\right)\left(\sqrt{v_{1}}+\sqrt{v_{2}}\right)^{2}}+1\right)
\end{aligned}
$$

with $v_{k} \equiv \operatorname{Var}\left(Z_{k}\right)=P_{2}+a_{k}^{2} P_{1}+2 a_{k} \rho \sqrt{P_{1} P_{u}}+1$.

Proof: As argued previously, the optimal DPC parameter for the common interference is $\gamma_{\text {opt }}=\frac{P_{v}}{\left(P_{v}+1\right)}$ while the optimal $\alpha$ is given by:

$$
\alpha_{o p t, 12}=\frac{a_{2} \sqrt{v_{1}}+a_{1} \sqrt{v_{2}}}{\sqrt{v_{1}}+\sqrt{v_{2}}} \frac{P_{v}}{P_{v}+1} .
$$

The remaining is simple algebra and thus, omitted.

4) Multiple Description Dirty-Paper Coding: Using the inner bound $\mathcal{R}$ given in expression (83), we derive a multiple description coding based DPC scheme where we let:

$$
\begin{gathered}
V=X_{v}+\gamma X_{u}+\alpha X_{1}, \\
V_{k}=X_{p}+\gamma_{k} X_{u}+\alpha_{k} X_{1},
\end{gathered}
$$

in which we split the common description power $P_{v}$ into $P_{v}-x$ and $x$ and where: $X_{v} \sim$ $\mathcal{N}\left(0, P_{v}-x\right) \perp X_{p} \sim \mathcal{N}(0, x)$.

We further combine with these DPC codewords a time sharing argument where the two private descriptions are activated in different time slots. This yields a zero correlation cost:

$$
I\left(V_{1} ; V_{2} \mid V U X_{1} T\right)=0 .
$$


Corollary 4 (MD-DPC lower bound). The optimal private description yields the rate:

$$
\begin{aligned}
\max _{\alpha, \gamma} \min _{k \in[1: 2]} \max _{\alpha_{k}}\left[I\left(V V_{k} ; Z_{k} \mid T\right)\right. & \left.-I\left(V V_{k} ; U X_{1} \mid T\right)\right]=\frac{1}{2} \log _{2}\left(P_{v}+1\right) \\
& -\frac{1}{2} \log _{2}\left(\frac{P_{1}\left(P_{v}+\left(1-\rho^{2}\right) P_{u}+1\right)\left(a_{1}-a_{2}\right)^{2} P(x)}{\left(P_{v}+1\right)\left(\sqrt{v_{1}}+\sqrt{v_{2}}\right)^{2}}+\sqrt{x+1}\right)
\end{aligned}
$$

where $P(x) \equiv \frac{P_{v}-x}{\sqrt{x+1}}$.

Proof: The proof is similar to the optimization of the private descriptions in [13] and is thus omitted.

In the sequel, we wish to compare the relative behaviour of both inner bounds through analysing the behaviour of MD-DPC as function of $x$ (the case $x=0$ corresponds to CDDPC inner bound). We wish also to evaluate their gap to the best outer bound that we can characterize for such a setting.

5) An outer bound for the weak interference regime: We can write an outer bound for the weak interference regime inspired from the non-multicast CIFC.

Corollary 5 (Outer bound for the Gaussian weak interference regime). The capacity region of the Gaussian Multicast CIFC is included in the set of rate pairs satisfying:

$$
\begin{aligned}
& R_{1} \leq \frac{1}{2} \log _{2}\left(\frac{b^{2} P_{2}+P_{1}+2|b| \sqrt{(1-\eta) P_{1} P_{2}}+1}{b^{2} \eta P_{2}+1}\right) \\
& R_{2} \leq \frac{1}{2} \log _{2}\left(\eta P_{2}+1\right)
\end{aligned}
$$

for some arbitrary $\eta \in[0: 1]$.

Proof: The proof follows along the same lines as in [3] and results from Fano's inequality that implies:

$$
\begin{aligned}
& n R_{2} \leq I\left(X_{2}^{n} ; Z_{k}^{n} \mid W_{1} X_{1}^{n}\right)+n \epsilon_{n}, \\
& n R_{1} \leq I\left(W_{1} X_{1}^{n} ; Y^{n}\right)+n \epsilon_{n},
\end{aligned}
$$

which turns out to be a non-multicast setup since conditioning on $X_{1}$, all channels $Z_{k}$ share the same statistics. 


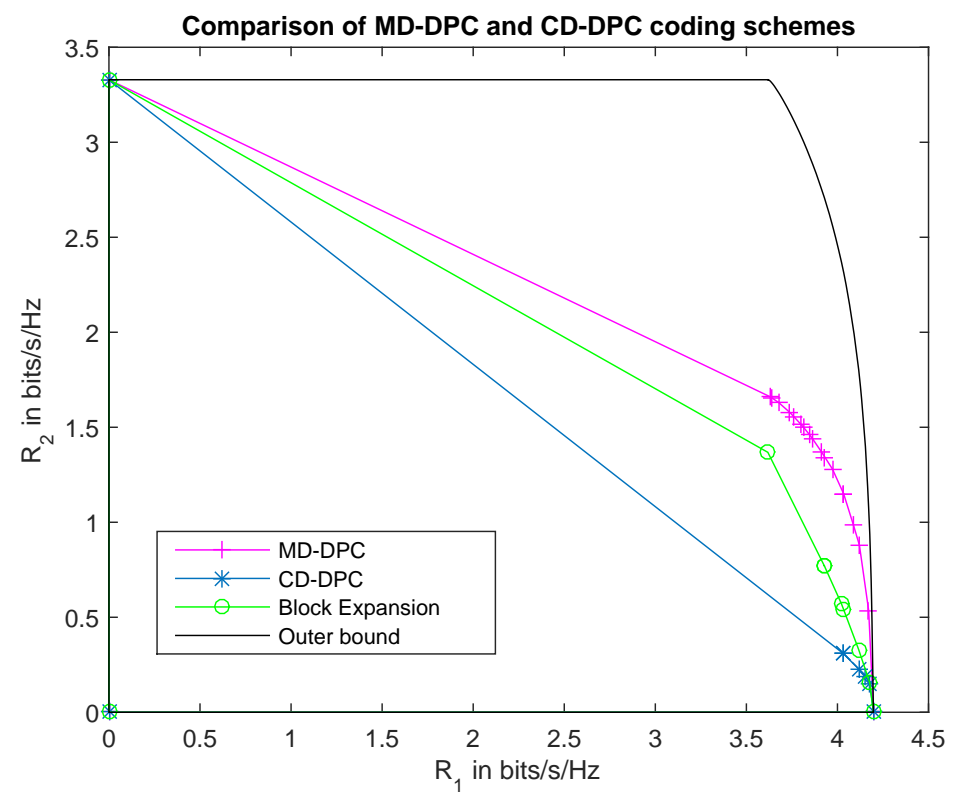

Fig. 9. Comparison of the bounds with: $P_{1}=3 P_{2}, a_{1}=.75, a_{2}=-.5, b=.1$.

6) Comparison of the inner and outer bounds: In the light of the results in [13], we can show by a simple function study that, the rate achieved by multiple description coding can be strictly increasing in $x$ and thus $x=0$ (CD-DPC) yields its minimum value. In Fig. 9, we plot the different inner bounds where we further combine the CD-DPC to a time-sharing argument, referred to as block expansion in [19]. It is clear then that the improved endowed by multiple description coding is strict and that it is becomes more relevant when the mismatch between the multicast channels $\left|a_{1}-a_{2}\right|$ and the primary user power $P_{1}$ increase.

We investigated the Multi-Secondary CIFC for which we characterize the capacity region in some interference regimes and show that, for the Gaussian weak interference case, a more evolved encoding scheme than the one allowed by the inner bound (5), is required to enhance the transmission rates.

\section{COMments AND Discussion}

\section{Anecdotic result}

In a recent paper by Vaezi [15], it is shown that the better cognitive decoding (BCD) introduced in [8] is nothing but the mere very weak interference (VWI) regime and that the new capacity 
result in this regime is an equivalent formulation of the VWI capacity region. However, the astonishing result in the work of Vaezi lies in proving that the very strong interference (VSI) regime is contained in the VWI regime for finite alphabets suggesting that apparently contradictory regimes from a conceptual point of view, are in fact equivalent. Later on, we clarify how the present work does not fall in such a triviality be it even for finite alphabet settings.

A crucial remark here is that the claim of Vaezi that the class of probability distribution that verify (VSI) falls into the class of (VWI) or equivalently, the (BCD), does not hold in the multicast setting. Let us first review this claim of triviality by Vaezi. The three regimes are given by the following conditions:

$$
\begin{aligned}
& \text { (VWI), } \forall P_{U X_{1} X_{2}}:\left\{\begin{array}{c}
I\left(U ; Y \mid X_{1}\right) \leq I\left(U ; Z \mid X_{1}\right), \\
I\left(X_{1} ; Y\right) \leq I\left(X_{1} ; Z\right),
\end{array}\right. \\
& \text { (BCD), } \forall P_{U X_{1} X_{2}}: I\left(U X_{1} ; Y\right) \leq I\left(U X_{1} ; Z\right), \\
& \text { (VSI), } \forall P_{X_{1} X_{2}}:\left\{\begin{array}{c}
I\left(X_{1} X_{2} ; Y\right) \leq I\left(X_{1} X_{2} ; Z\right), \\
I\left(X_{2} ; Z \mid X_{1}\right) \leq I\left(X_{2} ; Y \mid X_{1}\right) .
\end{array}\right.
\end{aligned}
$$

The key tool to show the equivalence between VWI and BCD is to notice that:

$$
\forall P_{U X_{1} X_{2}}, I\left(U X_{1} ; Y\right) \leq I\left(U X_{1} ; Z\right) \Rightarrow \forall P_{U X_{1} X_{2}}, I\left(U ; Y \mid X_{1}\right) \leq I\left(U ; Z \mid X_{1}\right) .
$$

Thus, since:

$$
\begin{array}{r}
I\left(X_{1} X_{2} ; Y\right) \leq I\left(X_{1} X_{2} ; Z\right) \text { and } I\left(X_{2} ; Z \mid X_{1}\right) \leq I\left(X_{2} ; Y \mid X_{1}\right) \\
\Rightarrow \forall P_{U}, I\left(U X_{1} ; Y\right) \leq I\left(U X_{1} ; Z\right),
\end{array}
$$

which means that VSI implies BCD which in turn is equivalent to VWI.

However, e.g. in the multi-primary setting, the distinct regimes are defined by:

$$
\begin{aligned}
\text { (VWI) }, \forall P_{U X_{1} X_{2}}:\left\{\begin{aligned}
\forall j \in[1: N], \quad I\left(U ; Y_{j} \mid X_{1}\right) & \leq I\left(U ; Z \mid X_{1}\right), \\
\min _{j \in[1: N]} I\left(U X_{1} ; Y_{j}\right) & \leq I\left(U X_{1} ; Z\right),
\end{aligned}\right. \\
\text { (VSI), } \forall P_{X_{1} X_{2}}:\left\{\begin{aligned}
\forall j \in[1: N], \quad I\left(X_{2} ; Z \mid X_{1}\right) & \leq I\left(X_{2} ; Y_{j} \mid X_{1}\right), \\
\min _{j \in[1: N]} I\left(X_{1} X_{2} ; Y_{j}\right) & \leq I\left(X_{1} X_{2} ; Z\right) .
\end{aligned}\right.
\end{aligned}
$$

It is clear when there is only one primary channel output, i.e., $N=1$, that what we recover is the BCD and the VSI regime. However, when $N>1$, there is no evidence why the VSI should 
be included in the VWI since: VSI implies $\min _{j \in[1: N]} I\left(U X_{1} ; Y_{j}\right) \leq I\left(U X_{1} ; Z\right)$, but the other constraint of VWI can not be implied since it is too strict:

$$
\text { VSI } \nRightarrow, \forall j \in[1: N] \quad I\left(U ; Y_{j} \mid X_{1}\right) \leq I\left(U ; Z \mid X_{1}\right) .
$$

Thus, VSI cannot imply VWI for all classes of multicast CIFC.

\section{A. Future line of work}

The analysis we made in this paper, for the Multi-Primary CIFC and Multi-Secondary CIFC, would serve as a baseline to further study the Multicast CIFC with arbitrary numbers of primary and secondary users. We emphasize here, however, that this constitutes a challenging task, for two main reasons: one that arises from the fact that the Multi-cast CIFC is even more general than the Compound Broadcast channel, for which only Degrees of Freedom are well understood to date, and the other one that stems from the fact that defining more general interference regimes becomes quickly intractable due to the multiple combinations of users.

Yet, introducing the ideas of Interference Decoding and Multiple Description coding will clearly be of crucial importance when dealing with more general Multicast CIFC settings and thus, a thoughtful combination of the two schemes seems to be needed for such settings.

\section{APPENDIX A}

\section{BASIC PROPERTIES AND RESULTS}

This appendix provides basic notions on some concepts used through this paper. Following definition in [20], we use in this paper strongly typical sets and the so-called delta-convention. Some useful facts are recalled here.

Definition 1. For any sequence $x^{n} \in \mathcal{X}^{n}$ and any symbol $a \in \mathcal{X}$, notation $N\left(a \mid x^{n}\right)$ stands for the number of occurrences of a in $x^{n}$.

Definition 2. A sequence $x^{n} \in \mathcal{X}^{n}$ is called (strongly) $\delta$-typical w.r.t. $X$ (or simply typical if the context is clear) if

$$
\left|\frac{1}{n} N\left(a \mid x^{n}\right)-P_{X}(a)\right| \leq \delta, \text { for each } a \in \mathcal{X},
$$

and $N\left(a \mid x^{n}\right)=0$ for each $a \in \mathcal{X}$ such that $P_{X}(a)=0$. The set of all such sequences is denoted by $T_{\delta}^{n}(X)$. 
Definition 3. Let $x^{n} \in \mathcal{X}^{n}$. A sequence $y^{n} \in \mathcal{Y}^{n}$ is called (strongly) $\delta$-typical (w.r.t. $Y$ ) given $x^{n}$ if

$$
\left|\frac{1}{n} N\left(a, b \mid x^{n}, y^{n}\right)-\frac{1}{n} N\left(a \mid x^{n}\right) P_{Y \mid X}(b \mid a)\right| \leq \delta \text { for each } a \in \mathcal{X}, b \in \mathcal{Y},
$$

and, $N\left(a, b \mid x^{n}, y^{n}\right)=0$ for each $a \in \mathcal{X}, b \in \mathcal{Y}$ such that $P_{Y \mid X}(b \mid a)=0$. The set of all such sequences is denoted by $T_{\delta}^{n}\left(Y \mid x^{n}\right)$.

Delta-convention [20]: For any sets $\mathcal{X}, \mathcal{Y}$, there exists a sequence $\left\{\delta_{n}\right\}_{n \in \mathbb{N}^{*}}$ such that lemmas below hold $\rrbracket^{1}$ From now on, typical sequences are understood with $\delta=\delta_{n}$. Typical sets are still denoted by $T_{\delta}^{n}(\cdot)$.

Lemma 1 ([20, Lemma 1.2.12]). There exists a sequence $\eta_{n} \underset{n \rightarrow \infty}{\longrightarrow} 0$ such that

$$
P_{X}^{n}\left(T_{\delta}^{n}(X)\right) \geq 1-\eta_{n}
$$

Lemma 2 (Joint typicality lemma [21]). There exists a sequence $\eta_{n} \underset{n \rightarrow \infty}{\longrightarrow} 0$ such that

$$
\left|-\frac{1}{n} \log P_{Y}^{n}\left(T_{\delta}^{n}\left(Y \mid x^{n}\right)\right)-I(X ; Y)\right| \leq \eta_{n}, \text { for each } x^{n} \in T_{\delta}^{n}(X) .
$$

Lemma 3 (Csiszár \& Körner's sum-identity [22, Lemma 7]). Consider two random sequences $X^{n}$ and $Y^{n}$ and a random variable C. The following identity holds:

$$
\sum_{i=1}^{n} I\left(Y_{i+1}^{n} ; X_{i} \mid C X^{i-1}\right)=\sum_{j=1}^{n} I\left(X^{j-1} ; Y_{j} \mid C Y_{j+1}^{n}\right) .
$$

\section{APPENDIX B}

\section{Proof of ACHIEVABILITY For the Multicast CIFC}

All the channel outputs in the Multicast setting are to be treated in a similar manner, thus, we show the achievability only with one primary channel output $Y$.

\section{Rate splitting:}

The message $W_{1}$ is split in two parts $W_{01}$ of rate $R_{01}$ that is intended to be decoded by all users and a private part $W_{11}$ that is intended to be decoded only by user $Y$.

Codebook generation: At source 1: First generate $2^{n R_{01}}$ sequences $q_{1}^{n}\left(w_{01}\right)$ following $\prod_{i=1}^{n} P_{Q_{1}}\left(q_{1, i}\left(w_{01}\right)\right)$. For each sequence $q_{1}^{n}\left(w_{01}\right)$, generate $2^{n R_{11}}$ sequences $x_{1}^{n}\left(w_{11}\right)$ following $\prod_{i=1}^{n} P_{X_{1} \mid Q_{1}}\left(x_{1, i}\left(w_{11}, w_{01}\right)\right)$.

\footnotetext{
${ }^{1}$ As a matter of fact, $\delta_{n} \rightarrow 0$ and $\sqrt{n} \delta_{n} \rightarrow \infty$ as $n \rightarrow \infty$.
} 
At Source 2: For each sequence $q_{1}^{n}\left(w_{01}\right)$, generate $2^{n\left(T_{02}\right)}$ sequences $q^{n}\left(w_{01}, s_{02}\right)$ following $\prod_{i=1}^{n} P_{Q \mid Q_{1}}\left(q_{i}\left(w_{01}, s_{02}\right)\right)$ and throw them in $2^{n R_{02}}$ bins $B_{0}^{n}\left(w_{01}, w_{02}\right)$. For each sequence $q^{n}\left(w_{01}, s_{02}\right)$, generate $2^{n T_{11}}$ sequences $u^{n}\left(s_{11}, w_{01}, s_{02}\right)$ and $2^{n T_{22}}$ sequences $v^{n}\left(s_{22}, w_{01}, s_{02}\right)$ following $\prod_{i=1}^{n} P_{U \mid Q Q_{1}}\left(u_{i}\left(s_{11}, w_{01}, s_{02}\right)\right)$ and $\prod_{i=1}^{n} P_{V \mid Q Q_{1}}\left(v_{i}\left(s_{22}, w_{01}, s_{02}\right)\right)$ and throw them respectively in $2^{n R_{11}}$ bins $B_{1}^{n}\left(w_{11}, w_{01}, s_{02}\right)$ and $2^{n R_{22}}$ bins $B_{2}^{n}\left(w_{22}, w_{01}, s_{02}\right)$.

Encoding: The encoder 1 sends $x_{1}^{n}\left(w_{11}, w_{01}\right)$. The encoder 2 finds in bin $B_{0}^{n}\left(w_{01}, w_{02}\right)$ a sequence indexed by $s_{02}$ such that:

$$
\left(x_{1}^{n}\left(w_{11}, w_{01}\right), q_{1}^{n}\left(w_{01}\right), q^{n}\left(w_{01}, s_{02}\right)\right) \in \mathcal{T}_{\left[Q Q_{1} X_{1}\right]}^{(n)} .
$$

Then, it looks in the product bin $B_{1}^{n}\left(w_{22}, w_{01}, s_{02}\right) \times B_{2}^{n}\left(w_{22}, w_{01}, s_{02}\right)$ for a couple of sequences such that:

$$
\left(x_{1}^{n}\left(w_{11}, w_{01}\right), q_{1}^{n}\left(w_{01}\right), q^{n}\left(w_{01}, s_{02}\right), u^{n}\left(s_{11}, w_{01}, s_{02}\right), v^{n}\left(s_{22}, w_{01}, s_{02}\right)\right) \in \mathcal{T}_{\left[Q Q_{1} U V X_{1}\right]}^{(n)}
$$

It then sends a codeword $x_{2}^{n}\left(s_{11}, s_{22}, w_{01}, s_{02}\right)$.

The encoding will be flawless if the following inequalities hold:

$$
\begin{gathered}
T_{02}-R_{02} \geq I\left(X_{1} ; Q \mid Q_{1}\right) \\
T_{11}-R_{11} \geq I\left(U ; X_{1} \mid Q_{1} Q\right) \\
T_{22}-R_{22} \geq I\left(V ; X_{1} \mid Q_{1} Q\right) \\
T_{11}-R_{11}+T_{22}-R_{22} \geq I\left(U ; V \mid Q_{1} Q\right)+I\left(U V ; X_{1} \mid Q_{1} Q\right) .
\end{gathered}
$$

Decoding: Receiver 2 decodes simultaneously the indices $\left(w_{01}, s_{02}, s_{22}\right)$ while decoder 1 decodes simultaneously $\left(w_{01}, s_{02}, w_{11}, s_{11}\right)$. The probability of error can be made arbitrarily small if the following inequalities hold:

$$
\begin{aligned}
T_{22} & \leq I\left(V ; Z \mid Q Q_{1}\right) \\
T_{02}+T_{22} & \leq I\left(Q V ; Z \mid Q_{1}\right), \\
R_{01}+T_{02}+T_{22} & \leq I\left(Q_{1} Q V ; Z\right), \\
T_{11} & \leq I\left(X_{1} U ; Y \mid Q\right)+I\left(Q U ; X_{1} \mid Q_{1}\right), \\
T_{02}+T_{11} & \leq I\left(X_{1} Q U ; Y \mid Q_{1}\right)+I\left(Q U ; X_{1} \mid Q_{1}\right), \\
R_{01}+T_{02}+T_{11} & \leq I\left(Q_{1} X_{1} Q U ; Y\right)+I\left(Q U ; X_{1} \mid Q_{1}\right) .
\end{aligned}
$$


Fourrier Motzkin Elimination: After running FME on binning rates $T_{02}, T_{11}, T_{22}$ and on rate splitting parameters $R_{01}$ and $R_{02}$, we end up with the rate region in Theorem 1 .

Multicast setting: All channel output $Y_{j}, j \in[1: N]$ perform all the same decoding strategy, thus, the rate region can be written similarly obtained replacing $Y$ with the minimum over all channel outputs $Y_{j}$, where $j \in[1: N]$. The same applied for all users $Z_{k}, k \in[1: M]$.

\section{APPENDIX C}

\section{Multi-Primary Very STRONG INTERFERENCE CAPACITY REgion}

The proof of converse follows from that the strong interference condition (16) implies that for all $P_{U}$ s.t $U \odot\left(X_{1}, X_{2}\right) \odot\left(Z, Y_{1}, \cdots, Y_{N}\right)$ :

$$
\forall j \in[1: N], \quad I\left(X_{2}^{n} ; Z^{n} \mid X_{1}^{n} U\right) \leq I\left(X_{2}^{n} ; Y_{j}^{n} \mid X_{1}^{n} U\right) .
$$

To see this, note the following:

$$
\begin{aligned}
I\left(X_{2}^{n} ; Z^{n} \mid X_{1}^{n} U\right) & -I\left(X_{2}^{n} ; Y_{j}^{n} \mid X_{1}^{n} U\right) \\
& =\sum_{i=1}^{n}\left[I\left(X_{2}^{n} ; Z_{i} \mid Z^{i-1} X_{1}^{n} U\right)-I\left(X_{2}^{n} ; Y_{j, i} \mid Y_{j, i+1}^{n} X_{1}^{n} U\right)\right] \\
& \stackrel{(a)}{=} \sum_{i=1}^{n}\left[I\left(X_{2}^{n} ; Z_{i} \mid Z^{i-1} Y_{j, i+1}^{n} X_{1}^{n} U\right)-I\left(X_{2}^{n} ; Y_{j, i} \mid Z^{i-1} Y_{j, i+1}^{n} X_{1}^{n} U\right)\right] \\
& \stackrel{(b)}{=} \sum_{i=1}^{n}\left[I\left(X_{2, i} ; Z_{i} \mid Z^{i-1} Y_{j, i+1}^{n} X_{1}^{n} U\right)-I\left(X_{2, i} ; Y_{j, i} \mid Z^{i-1} Y_{j, i+1}^{n} X_{1}^{n} U\right)\right] \\
& \stackrel{(c)}{\geq} 0
\end{aligned}
$$

where $(a)$ is a consequence of Csiszár \& Körner sum-identity applied twice as follows:

$$
\begin{aligned}
& \sum_{i=1}^{n}\left[I\left(X_{2}^{n} ; Z_{i} \mid Z^{i-1} X_{1}^{n} U\right)-I\left(X_{2}^{n} ; Y_{j, i} \mid Y_{j, i+1}^{n} X_{1}^{n} U\right)\right] \\
= & \sum_{i=1}^{n}\left[I\left(Y_{j, i+1}^{n} X_{2}^{n} ; Z_{i} \mid Z^{i-1} X_{1}^{n} U\right)-I\left(Z^{i-1} X_{2}^{n} ; Y_{j, i} \mid Y_{j, i+1}^{n} X_{1}^{n} U\right)\right] \\
= & \sum_{i=1}^{n}\left[I\left(X_{2}^{n} ; Z_{i} \mid Z^{i-1} Y_{j, i+1}^{n} X_{1}^{n} U\right)-I\left(X_{2}^{n} ; Y_{j, i} \mid Z^{i-1} Y_{j, i+1}^{n} X_{1}^{n} U\right)\right]
\end{aligned}
$$

and $(b)$ is a consequence of the following Markov Chain:

$$
\left(X_{2}^{i-1}, X_{2, i+1}^{n}\right) \odot\left(X_{1, i}, X_{2, i}, X_{1}^{i-1}, X_{1, i+1}^{n}, Z^{i-1}, Y_{j, i+1}^{n}\right) \odot\left(Y_{j, i}, Z_{j, i}\right)
$$


for all $i=[1: n]$, and finally $(c)$ is a consequence of the strong interference condition (16).

Thus, one can write that:

$$
\begin{aligned}
n\left(R_{2}-\epsilon_{n}\right) & \leq I\left(W_{2} ; Z^{n} \mid W_{1}\right) \\
& \leq I\left(X_{2}^{n} W_{2} ; Z^{n} \mid X_{1}^{n} W_{1}\right) \\
& \leq \sum_{i=1}^{n} I\left(X_{2}^{n} W_{2} ; Z_{i} \mid Z^{i-1} X_{1}^{n} W_{1}\right) \\
& =\sum_{i=1}^{n}\left[H\left(Z_{i} \mid Z^{i-1} X_{1}^{n} W_{1}\right)-H\left(Z_{i} \mid Z^{i-1} X_{2}^{n} X_{1}^{n} W_{2} W_{1}\right)\right] \\
& \leq \sum_{i=1}^{n}\left[H\left(Z_{i} \mid X_{1, i}\right)-H\left(Z_{i} \mid X_{2, i} X_{1, i}\right)\right] \\
& =\sum_{i=1}^{n} I\left(X_{2, i} ; Z_{i} \mid X_{1, i}\right) .
\end{aligned}
$$

Moreover, we can also write a sum-rate:

$$
\begin{aligned}
n\left(R_{1}+R_{2}-\epsilon_{n}\right) & \leq I\left(W_{1} ; Y_{j}^{n}\right)+I\left(W_{2} ; Z^{n} \mid W_{1}\right) \\
& \leq I\left(W_{1} X_{1}^{n} ; Y_{j}^{n}\right)+I\left(X_{2}^{n} ; Z^{n} \mid X_{1}^{n} W_{1}\right) \\
& \stackrel{(a)}{\leq} I\left(W_{1} X_{1}^{n} ; Y_{j}^{n}\right)+I\left(X_{2}^{n} ; Y_{j}^{n} \mid X_{1}^{n} W_{1}\right) \\
& =\sum_{i=1}^{n} I\left(X_{1, i} X_{2, i} ; Y_{j, i}\right),
\end{aligned}
$$

where $(a)$ is a consequence of inequality (122). The proof of the outer bound follows as usually by defining a time-sharing random variable $Q$ taking values on $[1: n]$ with uniform probability.

\section{APPENDIX D}

\section{MULTI-PRIMARY VERY WeAK INTERFERENCE CAPACITY REGION}

Consider the N-multicast CIFC under the weak interference condition given by (26). In order to show the converse to the region given by

$$
\left\{\begin{array}{l}
R_{1} \leq \min _{j \in[1: N]} I\left(U X_{1} ; Y_{j}\right), \\
R_{2} \leq I\left(X_{2} ; Z \mid X_{1} U\right),
\end{array}\right.
$$

for some arbitrarily dependent variables $\left(U, X_{1}, X_{2}\right)$ s.t $U \odot\left(X_{1}, X_{2}\right) \multimap\left(Z, Y_{1}, \cdots, Y_{N}\right)$, let $j \in[1: N]$. We have that from Fano's inequality:

$$
n\left(R_{2}-\epsilon_{n}\right) \leq I\left(W_{2} ; Z^{n} \mid W_{1} X_{1}^{n}\right)
$$




$$
\begin{aligned}
& \leq I\left(X_{2}^{n} ; Z^{n} \mid W_{1} X_{1}^{n}\right) \\
& =\sum_{i=1}^{n} I\left(X_{2}^{n} ; Z_{i} \mid Z^{i-1} W_{1} X_{1}^{n}\right) \\
& \leq \sum_{i=1}^{n} I\left(X_{2, i} ; Z_{i} \mid U_{i} X_{1, i}\right)
\end{aligned}
$$

where we defined $U_{i} \equiv\left(W_{1}, X_{1}^{i-1}, X_{1, i+1}^{n}, Z^{i-1}\right)$. Now on the other side,

$$
\begin{aligned}
n\left(R_{1}-\epsilon_{n}\right) & \leq I\left(W_{1} X_{1}^{n} ; Y_{j}^{n}\right) \\
& =\sum_{i=1}^{n} I\left(W_{1} X_{1}^{n} Y_{j}^{i-1} ; Y_{j, i}\right) .
\end{aligned}
$$

The main idea here is that, we can upper bound this expression letting $Z^{i-1}$ replace $Y_{j}^{i-1}$, i.e.,

$$
\forall i \in[1: n] \quad, \quad I\left(W_{1} X_{1}^{n} Y_{j}^{i-1} ; Y_{j, i}\right) \leq I\left(W_{1} X_{1}^{n} Z^{i-1} ; Y_{j, i}\right)
$$

This is due to the conditional less-noisiness of $Y_{j}$ compared to $Z$ given $X_{1}$ in 26) and it is easy to check that by following similar lines as in [18]:

$$
\begin{aligned}
I\left(W_{1} X_{1}^{n} Z^{i-1} ; Y_{j, i}\right) & -I\left(W_{1} X_{1}^{n} Y_{j}^{i-1} ; Y_{j, i}\right) \\
& =I\left(Z^{i-1} ; Y_{j, i} \mid W_{1} X_{1}^{n}\right)-I\left(Y_{j}^{i-1} ; Y_{j, i} \mid W_{1} X_{1}^{n}\right) \\
& =\sum_{r=1}^{i-1}\left[I\left(Y_{j}^{r-1} Z_{r}^{i-1} ; Y_{j, i} \mid W_{1} X_{1}^{n}\right)-I\left(Y_{j}^{r} Z_{r+1}^{i-1} ; Y_{j, i} \mid W_{1} X_{1}^{n}\right)\right] \\
& =\sum_{r=1}^{i-1}\left[I\left(Z_{r} ; Y_{j, i} \mid Y_{j}^{r-1} Z_{r+1}^{i-1} W_{1} X_{1}^{n}\right)-I\left(Y_{j, r} ; Y_{j, i} \mid Y_{j}^{r-1} Z_{r+1}^{i-1} W_{1} X_{1}^{n}\right)\right] \\
& =\sum_{r=1}^{i-1}\left[I\left(Z_{r} ; Y_{j, i} \mid X_{1, r} Y_{j}^{r-1} Z_{r+1}^{i-1} W_{1} X_{1, r+1}^{n} X_{1}^{r-1}\right)\right. \\
& \left.-I\left(Y_{j, r} ; Y_{j, i} \mid X_{1, r} Y_{j}^{r-1} Z_{r+1}^{i-1} W_{1} X_{1, r+1}^{n} X_{1}^{r-1}\right)\right] \\
& (a)
\end{aligned}
$$

The condition in 26 implies that for all $r \in[1: i-1]$ and all $V$ :

$$
I\left(U ; Y_{r} \mid V X_{1, r}\right) \leq I\left(U ; Z_{r} \mid V X_{1, r}\right)
$$

Letting $U \equiv Y_{j, i}$ and $V \equiv\left(Y_{j}^{r-1}, Z_{r+1}^{i-1}, W_{1}, X_{1, r+1}^{n}, X_{1}^{r-1}\right)$, the claim in $(a)$ is proved. Thus,

$$
n R_{1}-n \epsilon_{n} \leq \sum_{i=1}^{n} I\left(W_{1} X_{1}^{n} Y_{j}^{i-1} ; Y_{j, i}\right)
$$




$$
\leq \sum_{i=1}^{n} I\left(W_{1} X_{1}^{n} Z^{i-1} ; Y_{j, i}\right)
$$

which completes the proof.

\section{APPENDIX E}

\section{MULTI-PRIMARY MiXed WEAK/STRONG INTERFERENCE CAPACITY REGION}

For the converse part, we can write that:

$$
\begin{gathered}
n R_{1} \leq \min _{j \in \mathcal{W}} \sum_{i=1}^{n} I\left(U_{i} X_{1, i} ; Y_{j, i}\right), \\
n R_{2} \leq \sum_{i=1}^{n} I\left(X_{2, i} ; Z_{i} \mid U_{i} X_{1, i}\right), \\
n\left(R_{1}+R_{2}\right) \leq \min _{j \in \mathcal{S}} \sum_{i=1}^{n} I\left(X_{1, i} X_{2, i} ; Y_{j, i}\right),
\end{gathered}
$$

where $U_{i}=\left(W_{1}, X_{1, i+1}^{n}, X_{1}^{i-1}, Z^{i-1}\right)$ for all $i=[1: n]$.

As for the achievability part, we consider the codebook construction in Appendix B, and let $Q_{1}=\emptyset$ and $Q=U$. We can summarize the encoding constraints as follows:

$$
\begin{aligned}
& T_{1}-R_{1} \geq I\left(U ; X_{1}\right), \\
& T_{2}-R_{2} \geq I\left(V ; X_{1} \mid U\right) .
\end{aligned}
$$

As for the decoding constraints, user $Z$ decodes the signal $U$ and $X_{1}$ non-uniquely, finding the unique $s_{2}$ such that for some $w_{1}$ and $s_{1}$ :

$$
\left(u^{n}\left(s_{1}\right), x_{1}^{n}\left(w_{1}\right), v^{n}\left(s_{1}, s_{2}\right), y_{j}^{n}\right) \in T_{\delta}^{n}\left(U X_{1} V Z\right),
$$

where $u^{n}\left(s_{1}\right)$ is in the bin defined by $s_{1}$. Thus, we end up with the constraints:

$$
\begin{aligned}
T_{2} & \leq I\left(V ; Z \mid X_{1} U\right)+I\left(V ; X_{1} \mid U\right), \\
T_{1}+T_{2} & \leq I\left(X_{1} U V ; Z\right)+I\left(U V ; X_{1}\right) .
\end{aligned}
$$

On the other side, the users $Y_{j}$ can choose between two decoding strategies:

- Not decoding interference, i.e., finding the unique $w_{1}$ for which:

$$
\left(u^{n}\left(s_{1}\right), x_{1}^{n}\left(w_{1}\right), y_{j}^{n}\right) \in T_{\delta}^{n}\left(U X_{1} Y_{j}\right),
$$


where $u^{n}\left(s_{1}\right) \in B_{1}^{n}\left(w_{1}\right)$ is in the bin defined by $w_{1}$. This yields the following constraint:

$$
T_{1} \leq I\left(U X_{1} ; Y_{j}\right)+I\left(U ; X_{1}\right) .
$$

- Decoding interference non-uniquely, finding the unique $w_{1}$ such that for some $s_{2}$,

$$
\left(u^{n}\left(s_{1}\right), x_{1}^{n}\left(w_{1}\right), v^{n}\left(s_{1}, s_{2}\right), y_{j}^{n}\right) \in T_{\delta}^{n}\left(U X_{1} V Y_{j}\right) .
$$

This results in the constraint:

$$
T_{1}+T_{2} \leq I\left(U X_{1} V ; Y_{j}\right)+I\left(V U ; X_{1}\right) .
$$

One then can write an achievable inner bound with all possible combinations of decoding choices of each of the users $Y_{j}$.

Using this idea, we let the group of users in strong interference decode interference as well, and we let the users in weak interference decode only their intended signals $U$ and $X_{1}$. The resulting set of constraints is given by:

$$
\left\{\begin{aligned}
T_{1} & \leq \min _{j \in \mathcal{W}} I\left(U X_{1} ; Y_{j}\right)+I\left(U ; X_{1}\right), \\
T_{2} & \leq I\left(V ; Z \mid X_{1} U\right)+I\left(V ; X_{1} \mid U\right), \\
T_{1}+T_{2} & \leq I\left(X_{1} U V ; Z\right)+I\left(U V ; X_{1}\right), \\
T_{1}+T_{2} & \leq \min _{j \in \mathcal{S}} I\left(U X_{1} V ; Y_{j}\right)+I\left(U V ; X_{1}\right) .
\end{aligned}\right.
$$

Running FME on the resulting rate region yields:

$$
\left\{\begin{aligned}
R_{1} & \leq \min _{j \in \mathcal{W}} I\left(U X_{1} ; Y_{j}\right), \\
R_{2} & \leq I\left(V ; Z \mid X_{1} U\right), \\
R_{1}+R_{2} & \leq I\left(X_{1} U V ; Z\right), \\
R_{1}+R_{2} & \leq \min _{j \in \mathcal{S}} I\left(U X_{1} V ; Y_{j}\right) .
\end{aligned}\right.
$$

Letting $V=X_{2}$, we end up with the following achievable region:

$$
\left\{\begin{aligned}
R_{1} & \leq \min _{j \in \mathcal{W}} I\left(U X_{1} ; Y_{j}\right), \\
R_{2} & \leq I\left(X_{2} ; Z \mid X_{1} U\right) \\
R_{1}+R_{2} & \leq I\left(X_{1} X_{2} ; Z\right) \\
R_{1}+R_{2} & \leq \min _{j \in \mathcal{S}} I\left(X_{1} X_{2} ; Y_{j}\right)
\end{aligned}\right.
$$

with the strong interference or the weak interference condition, we can show that the sum-rate $R_{1}+R_{2} \leq I\left(X_{1} X_{2} ; Z\right)$ is redundant. This completes the proof of achievability. 


\section{APPENDIX F \\ Proof OF Theorem 5: Gaussian Multi-Primary CIFC}

\section{A. Very Strong Interference}

We start with the achievability part. Consider the following coding scheme:

$$
\left(X_{1}, X_{2}\right) \sim \mathcal{N}\left(\mathbf{0}, \Sigma_{X_{1} X_{2}}\right), \Sigma_{X_{1} X_{2}}=\left[\begin{array}{cc}
P_{1} & \rho \sqrt{P_{1} P_{2}} \\
\rho \sqrt{P_{1} P_{2}} & P_{2}
\end{array}\right] .
$$

Then, letting $j \in[1: N]$ :

$$
\begin{aligned}
& I\left(X_{1} X_{2} ; Y_{j}\right)=\frac{1}{2} \log _{2}\left(1+b_{j}^{2} P_{2}+P_{1}+2 b_{j} \rho \sqrt{P_{1} P_{2}}\right), \\
& I\left(X_{2} ; Z \mid X_{1}\right)=\frac{1}{2} \log _{2}\left(1+\left(1-\rho^{2}\right) P_{2}\right),
\end{aligned}
$$

which completes the proof of achievability.

As for the converse, following the same lines as the proof of the outer bound of Rini et al. [9], we can write the following outer bound as:

$$
\left\{\begin{aligned}
R_{1} & \leq \min _{j \in[1: N]} I\left(X_{1} X_{2} ; Y_{j}\right) \\
R_{2} & \leq I\left(X_{2} ; Z \mid X_{1}\right) \\
R_{1}+R_{2} & \leq \min _{j \in[1: N]} I\left(X_{1} X_{2} ; Y_{j}\right)+I\left(X_{2} ; Z \mid Y_{j}^{\prime} X_{1}\right) .
\end{aligned}\right.
$$

Similarly to the result of [9], we compute the optimal correlation coefficient between $Z$ and $Y_{j}^{\prime}$ conditioned on $X_{1}$. We obtain then, the following outer bound:

$$
\begin{aligned}
R_{2} & \leq \frac{1}{2} \log _{2}\left(1+\left(1-\rho^{2}\right) P_{2}\right), \\
R_{1} & \leq \frac{1}{2} \min _{j \in[1: N]} \log _{2}\left(1+b_{j}^{2} P_{2}+P_{1}+2 b_{j} \rho \sqrt{P_{1} P_{2}}\right), \\
R_{1}+R_{2} & \leq \frac{1}{2} \min _{j \in[1: N]}\left[\log _{2}\left(1+b_{j}^{2} P_{2}+P_{1}+2 b_{j} \rho \sqrt{P_{1} P_{2}}\right)\right. \\
& \left.+\frac{1}{2} \log _{2}^{+}\left(\frac{1+\left(1-\rho^{2}\right) P_{2}}{1+\left(1-\rho^{2}\right) P_{2} b_{j}^{2}}\right)\right],
\end{aligned}
$$

where $\log _{2}^{+}(x) \equiv \max \left\{0, \log _{2}(x)\right\}$.

Since $\left|b_{j}\right|>1$ for all $j \in[1: N]$, then the outer bound becomes equal to:

$$
\begin{aligned}
R_{2} & \leq \frac{1}{2} \log _{2}\left(1+\left(1-\rho^{2}\right) P_{2}\right), \\
R_{1}+R_{2} & \leq \frac{1}{2} \min _{j \in[1: N]} \log _{2}\left(1+b_{j}^{2} P_{2}+P_{1}+2 b_{j} \rho \sqrt{P_{1} P_{2}}\right),
\end{aligned}
$$

which proves our claim. 


\section{B. Weak Interference}

The achieavability follows from the achievable rate region:

$$
\left\{\begin{array}{l}
R_{1} \leq \min _{j \in[1: N]} I\left(X_{1} U ; Y_{j}\right), \\
R_{2} \leq I(V ; Z)-I\left(V ; X_{1} U\right),
\end{array}\right.
$$

obtained through the inner bound (5) by letting $Q_{1}=Q=\emptyset$ and considering only one of the resulting corner points.

The optimal coding scheme is then to let:

$$
\begin{aligned}
X_{2} & =X_{u}+X_{v} \quad, \quad X_{v} \sim \mathcal{N}\left(0, \eta P_{2}\right) \quad, \quad X_{u} \sim \mathcal{N}\left(0,(1-\eta) P_{2}\right) \\
U & =X_{u} \quad, \quad X_{1} \sim \mathcal{N}\left(0, P_{1}\right), \\
\left(X_{1}, X_{u}\right) & \sim \mathcal{N}\left(\mathbf{0}, \Sigma_{X_{1} X_{u}}\right), \quad \Sigma_{X_{1} X_{u}}=\left[\begin{array}{cc}
P_{1} & \rho \sqrt{P_{1}(1-\eta) P_{2}} \\
\rho \sqrt{P_{1}(1-\eta) P_{2}} & (1-\eta) P_{2}
\end{array}\right] \\
V & =X_{v}+\gamma\left(X_{u}+a X_{1}\right),
\end{aligned}
$$

where $|\rho| \leq 1$ and $\gamma$ is the optimal Dirty-Paper Coding parameter to precode against the interference $X_{u}+a X_{1}$ seen at user $Z$.

Thus, we obtain:

$$
\begin{aligned}
I\left(X_{u} X_{1} ; Y_{j}\right) & =h\left(Y_{j}\right)-h\left(Y_{j} \mid X_{u} X_{1}\right) \\
& =\frac{1}{2} \log _{2}\left(\frac{1+b_{j}^{2} P_{2}+P_{1}+2 b_{j} \rho \sqrt{(1-\eta) P_{1} P_{2}}}{1+b_{j}^{2} \eta P_{2}}\right),
\end{aligned}
$$

and

$$
I(V ; Z)-I\left(V ; U X_{1}\right)=I\left(X_{v} ; Z \mid X_{1} X_{u}\right)=\frac{1}{2} \log _{2}\left(1+\eta P_{2}\right) .
$$

N.B: It is because we can apply DPC techniques for the Gaussian case that we are able to relax the constraint of very weak interference to only weak interference. In the general case, it is not possible for user $Z$ to decode interference unless its resulting sum-rate is satisfied, i.e., $R_{1}+R_{2} \leq I\left(X_{1} X_{2} ; Z\right)$.

The parameter $\rho$ cannot be optimized for each instance of the primary channels $Y_{j}$ since the $b_{j}$ 's are not all compulsorily equal (in sign and module), leading us to the max min expression.

Hereafter, the converse proof. Let us start by writing

$$
n R_{2}=H\left(W_{2}\right)
$$




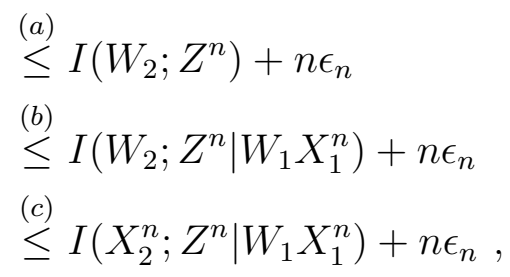

where $(a)$ is a consequence of Fano's inequality and $(b)$ follows from the fact that $W_{2}$ is independent of both $W_{1}$ and $X_{1}^{n}$, and $(c)$ results from the fact that the following Markov Chain holds $W_{2} \multimap\left(X_{2}^{n}, X_{1}^{n}, W_{1}\right) \multimap Z^{n}$.

Then, let $j \in[1: N]$, we have that:

$$
\begin{aligned}
n R_{1} & =H\left(W_{1}\right) \\
& \leq I\left(W_{1} ; Y_{j}^{n}\right)+n \epsilon_{n} \\
& \leq I\left(W_{1} X_{1}^{n} ; Y_{j}^{n}\right)+n \epsilon_{n} .
\end{aligned}
$$

Next, we bound the two resulting rates. Since,

$$
\frac{n}{2} \log _{2}(2 \pi e) \leq h\left(Z^{n} \mid W_{1} X_{1}^{n}\right) \leq \frac{n}{2} \log _{2}\left(P_{2}+1\right)+\frac{n}{2} \log _{2}(2 \pi e),
$$

then

$$
\exists \eta \in[0: 1] \quad \text { such that } h\left(Z^{n} \mid W_{1} X_{1}^{n}\right)=\frac{n}{2} \log _{2}\left(\eta P_{2}+1\right)+\frac{n}{2} \log _{2}(2 \pi e),
$$

and since,

$$
h\left(Z^{n} \mid X_{2}^{n} X_{1}^{n}\right)=\frac{n}{2} \log _{2}(2 \pi e),
$$

we can conclude that

$$
R_{2} \leq \frac{1}{n} I\left(X_{2}^{n} ; Z^{n} \mid W_{1} X_{1}^{n}\right)=\frac{1}{2} \log _{2}\left(\eta P_{2}+1\right) .
$$

Next, note that, with an abuse of notations:

$$
Y_{j}^{n} \mid X_{1}^{n}=b_{j} X_{2}^{n}+n_{2}^{n}=b_{j}\left(Z^{n} \mid X_{1}^{n}\right)+b_{j} \tilde{N}_{2}^{n},
$$

where $\tilde{N}_{2} \sim \mathcal{N}\left(0, b_{j}^{-2}-1\right)$.

Thus, we can write by the $n$-letter conditional EPI that:

$$
\begin{aligned}
h\left(Y_{j}^{n} \mid W_{1} X_{1}^{n}\right) & \geq \frac{n}{2} \log _{2}\left(2^{\frac{2}{n} h\left(b_{j} Z^{n} \mid W_{1} X_{1}^{n}\right)}+2^{\frac{2}{n} h\left(b_{j} \tilde{N}_{2}^{n}\right)}\right) \\
& =\frac{n}{2} \log _{2}\left[b_{j}^{2} 2^{\frac{2}{n} h\left(Z^{n} \mid W_{1} X_{1}^{n}\right)}+2 \pi e\left(1-b_{j}^{2}\right)\right]
\end{aligned}
$$




$$
\begin{aligned}
& =\frac{n}{2} \log _{2}\left[b_{j}^{2}\left(\eta P_{2}+1\right)+1-b_{j}^{2}\right]+\frac{n}{2} \log _{2}(2 \pi e) \\
& =\frac{n}{2} \log _{2}\left[b_{j}^{2} \eta P_{2}+1\right]+\frac{n}{2} \log _{2}(2 \pi e) .
\end{aligned}
$$

In addition, letting besides $\left(X_{1}, X_{2}\right)$ to have the following covariance matrix:

$$
K=\left[\begin{array}{cc}
P_{1} & \rho_{12} \sqrt{P_{1} P_{2}} \\
\rho_{12} \sqrt{P_{1} P_{2}} & P_{2}
\end{array}\right],
$$

we can combine with:

$$
\begin{aligned}
\frac{n}{2} \log _{2}\left[2 \pi e\left(b_{j}^{2} \eta P_{2}+1\right)\right] & \leq h\left(Y_{j}^{n} \mid W_{1} X_{1}^{n}\right) \\
& \leq h\left(Y_{j}^{n} \mid X_{1}^{n}\right) \leq \frac{n}{2} \log _{2}\left[2 \pi e\left(b_{j}^{2}\left(1-\rho_{12}^{2}\right) P_{2}+1\right)\right]
\end{aligned}
$$

thus,

$$
\eta \leq 1-\rho_{12}^{2}
$$

Finally, we let $\rho \in[-1: 1]$ such that

$$
\rho=\frac{\rho_{12}}{\sqrt{1-\eta}}
$$

to obtain:

$$
R_{1} \leq \frac{1}{2} \log _{2}\left(1+b_{j}^{2} \eta P_{2}+P_{1}+2 b_{j} \rho \sqrt{P_{1}(1-\eta) P_{2}}\right)-\frac{1}{2} \log _{2}\left(b_{j}^{2} \eta P_{2}+1\right),
$$

which concludes the proof.

\section{Mixed Weak/Very Strong Interference}

The proof of the achievability follows by evaluating the rate region given by:

$$
\left\{\begin{aligned}
R_{1} & \leq \min _{j \in \mathcal{W}} I\left(U X_{1} ; Y_{j}\right), \\
R_{2} & \leq I\left(X_{2} ; Z \mid U X_{1}\right), \\
R_{1}+R_{2} & \leq \min _{j \in \mathcal{S}} I\left(X_{1} X_{2} ; Y_{j}\right),
\end{aligned}\right.
$$

with the following coding scheme:

$$
\begin{aligned}
X_{2} & =X_{u}+X_{v} \quad, \quad X_{v} \sim \mathcal{N}\left(0, \eta P_{2}\right) \quad, \quad X_{u} \sim \mathcal{N}\left(0,(1-\eta) P_{2}\right) \\
U & =X_{u} \quad, \quad X_{1} \sim \mathcal{N}\left(0, P_{1}\right), \\
\left(X_{1}, X_{u}\right) & \sim \mathcal{N}\left(\mathbf{0}, \Sigma_{X_{1} X_{u}}\right), \Sigma_{X_{1} X_{u}}=\left[\begin{array}{cc}
P_{1} & \rho \sqrt{P_{1}(1-\eta) P_{2}} \\
\rho \sqrt{P_{1}(1-\eta) P_{2}} & (1-\eta) P_{2}
\end{array}\right]
\end{aligned}
$$


where $|\rho| \leq 1$. Then, we obtain:

$$
\begin{aligned}
I\left(X_{u} X_{1} ; Y_{j}\right) & =h\left(Y_{j}\right)-h\left(Y_{j} \mid X_{u} X_{1}\right) \\
& =\frac{1}{2} \log _{2}\left(\frac{1+b_{j}^{2} P_{2}+P_{1}+2 b_{j} \rho \sqrt{(1-\eta) P_{1} P_{2}}}{1+b_{j}^{2} \eta P_{2}}\right)
\end{aligned}
$$

and

$$
I\left(X_{2} ; Z \mid X_{1} X_{u}\right)=\frac{1}{2} \log _{2}\left(1+\eta P_{2}\right)
$$

Finally,

$$
I\left(X_{2} X_{1} ; Y_{j}\right)=\frac{1}{2} \log _{2}\left(1+b_{j}^{2} P_{2}+P_{1}+2 b_{j} \rho \sqrt{(1-\eta) P_{1} P_{2}}\right) .
$$

As for the outer bound, we can similarly to the weak and strong interference case, write that:

$$
\begin{aligned}
n R_{1} & \leq \min _{j \in \mathcal{W}} I\left(W_{1} X_{1}^{n} ; Y_{j}^{n}\right)+n \epsilon_{n}, \\
n R_{2} & \leq I\left(X_{2}^{n} ; Z^{n} \mid W_{1} X_{1}^{n}\right)+n \epsilon_{n}, \\
n\left(R_{1}+R_{2}\right) & \leq \min _{j \in \mathcal{S}} I\left(X_{1}^{n} X_{2}^{n} ; Y_{j}^{n}\right)+n \epsilon_{n} .
\end{aligned}
$$

In the same fashion again, define $\eta \in[0: 1]$ such that:

$$
h\left(Z^{n} \mid W_{1} X_{1}^{n}\right)=\frac{n}{2} \log _{2}\left(\eta P_{2}+1\right)+\frac{n}{2} \log _{2}(2 \pi e) .
$$

We can show that for all $j \in \mathcal{W}$,

$$
h\left(Y_{j}^{n} \mid W_{1} X_{1}^{n}\right) \geq \frac{n}{2} \log _{2}\left(\eta b_{j}^{2} P_{2}+1\right)+\frac{n}{2} \log _{2}(2 \pi e) .
$$

As for $h\left(Y_{j}^{n}\right)$ with $j \in[1: N]$, it is maximized when $\left(X_{1}, X_{2}\right)$ follow a joint Gaussian distribution with covariance matrix:

$$
K=\left[\begin{array}{cc}
P_{1} & \rho_{12} \sqrt{P_{1} P_{2}} \\
\rho_{12} \sqrt{P_{1} P_{2}} & P_{2}
\end{array}\right],
$$

where $\rho_{12} \in[-1: 1]$.

Now, it can be noticed that $\rho_{12}$ has to satisfy the inequality:

$$
\eta \leq 1-\rho_{12}^{2}
$$

Let $\rho \in[-1: 1]$ such that

$$
\rho=\frac{\rho_{12}}{\sqrt{1-\eta}}
$$

which yields an outer bound equasl to the claimed capacity region (52). 


\section{Coherent and non-coherent Interference}

Since the three claims have similar proofs, we only show the first one. The first part of the proof is trivial since the optimal $\rho$ is obtained by +1 if all channel coefficients $b_{j}$ are positive, and -1 otherwise. The second half of the claim can be proved by letting $C_{j}$ be the capacity region of the CIFC $\left(Z, Y_{j}\right)$ in weak interference. Assume that all $b_{j}$ 's are positive. We have that:

$$
\mathcal{C}_{j}(\eta):\left\{\begin{array}{l}
R_{1} \leq R_{1, j}(\eta) \equiv \frac{1}{2} \log _{2}\left(\frac{1+b_{j}^{2} P_{2}+P_{1}+2 b_{j} \sqrt{(1-\eta) P_{1} P_{2}}}{1+b_{j}^{2} \eta P_{2}}\right), \\
R_{2} \leq R_{2}(\eta) \equiv \frac{1}{2} \log _{2}\left(1+\eta P_{2}\right) .
\end{array}\right.
$$

We want to show that:

$$
\bigcap_{j=1}^{N} \bigcup_{\eta \in[0: 1]} \mathcal{C}_{j}(\eta)=\bigcup_{\eta \in[0: 1]} \mathcal{C}(\eta)
$$

We have that for all $j \in[1: N], \mathcal{C}(\eta) \subset \mathcal{C}_{j}(\eta)$, thus it is easy to prove the first inclusion:

$$
\bigcup_{\eta \in[0: 1]} \mathcal{C}(\eta) \subset \bigcap_{j=1}^{N} \bigcup_{\eta \in[0: 1]} \mathcal{C}_{j}(\eta)
$$

Now, to show the other inclusion, let $\left(R_{1}, R_{2}\right)$ be a rate pair in $\bigcap_{j=1}^{N} \bigcup_{\eta \in[0: 1]} \mathcal{C}_{j}(\eta)$. We need to show that $\left(R_{1}, R_{2}\right)$ lies in $\bigcup_{\eta \in[0: 1]} \mathcal{C}(\eta)$. Let $\eta_{1} \ldots \eta_{N}$ be $\mathrm{N}$ parameters such that:

$$
\left\{\begin{array}{l}
R_{1} \leq \min _{j \in[1: N]} R_{1, j}\left(\eta_{j}\right) \\
R_{2} \leq \min _{j \in[1: N]} R_{2}\left(\eta_{j}\right)
\end{array}\right.
$$

Note that, $R_{2}$ is increasing in $\eta$ while $R_{1, j}$ is decreasing in $\eta$. Thus, we have

$$
R_{2} \leq \min _{j \in[1: N]} R_{2}\left(\eta_{j}\right)=R_{2}\left(\min _{j \in[1: N]} \eta_{j}\right)
$$

And hence, since $R_{1, j}$ is decreasing in $\eta$, then:

$$
R_{1} \leq \min _{j \in[1: N]} R_{1, j}\left(\eta_{j}\right) \leq \min _{j \in[1: N]} R_{1, j}\left(\min _{j \in[1: N]} \eta_{j}\right)
$$

thus, setting $\eta \equiv \min _{j \in[1: N]} \eta_{j}$ allows $\left(R_{1}, R_{2}\right)$ to lie in the region $\bigcup_{\eta \in[0: 1]} \mathcal{C}(\eta)$. 


\section{APPENDIX G}

\section{Multi-SECONDARY VERY WEAK INTERFERENCE CAPAACITY REGION}

From Fano's inequality, we can write that:

$$
\begin{aligned}
n\left(R_{1}-\epsilon_{n}\right) & \leq I\left(W_{1} ; Y^{n}\right) \\
& \leq I\left(W_{1} X_{1}^{n} ; Y^{n}\right) \\
& \leq \sum_{i=1}^{n} I\left(W_{1} X_{1, i} X_{1,<i>} Y^{i-1} ; Y_{i}\right),
\end{aligned}
$$

where $X_{1,<i>} \equiv\left(X_{1}^{i-1}, X_{1, i+1}^{n}\right)$.

Similarly, for the other rate, let $k \in[1: M]$ :

$$
\begin{aligned}
n\left(R_{2}-\epsilon_{n}\right) \leq & I\left(X_{2}^{n} ; Z_{k}^{n} \mid W_{1} X_{1}^{n}\right) \\
= & \sum_{i=1}^{n} I\left(X_{2, i} ; Z_{k, i} \mid W_{1} X_{1, i} X_{1,<i>} Z_{k}^{i-1}\right) \\
= & \sum_{i=1}^{n}\left[I\left(X_{2, i} ; Z_{k, i} \mid W_{1} X_{1, i} X_{1,<i>}\right)\right. \\
& \left.\quad-I\left(Z_{k}^{i-1} ; Z_{k, i} \mid W_{1} X_{1, i} X_{1,<i>}\right)\right] \\
& \stackrel{(a)}{\leq} \sum_{i=1}^{n}\left[I\left(X_{2, i} ; Z_{k, i} \mid W_{1} X_{1, i} X_{1,<i>}\right)\right. \\
& \left.\quad-I\left(Y^{i-1} ; Z_{k, i} \mid W_{1} X_{1, i} X_{1,<i>}\right)\right] \\
= & \sum_{i=1}^{n} I\left(X_{2, i} ; Z_{k, i} \mid W_{1} X_{1, i} X_{1,<i>} Y^{i-1}\right)
\end{aligned}
$$

where $(a)$ is a result of (64) and is proved as follows. Let $i \in[1: n]$, we have that:

$$
\begin{aligned}
I\left(W_{1} X_{1}^{n} Z_{k}^{i-1} ; Y_{i}\right) & -I\left(W_{1} X_{1}^{n} Y^{i-1} ; Y_{i}\right) \\
& =I\left(Z_{k}^{i-1} ; Y_{i} \mid W_{1} X_{1}^{n}\right)-I\left(Y^{i-1} ; Y_{i} \mid W_{1} X_{1}^{n}\right) \\
& =\sum_{r=1}^{i-1}\left[I\left(Y^{r-1} Z_{k, r}^{i-1} ; Y_{i} \mid W_{1} X_{1}^{n}\right)-I\left(Y^{r} Z_{k, r+1}^{i-1} ; Y_{i} \mid W_{1} X_{1}^{n}\right)\right] \\
& =\sum_{r=1}^{i-1}\left[I\left(Z_{k, r} ; Y_{i} \mid X_{1, r} Y^{r-1} Z_{k, r+1}^{i-1} W_{1} X_{1, r+1}^{n} X_{1}^{r-1}\right)\right. \\
& \left.-I\left(Y_{r} ; Y_{i} \mid X_{1, r} Y^{r-1} Z_{k, r+1}^{i-1} W_{1} X_{1, r+1}^{n} X_{1}^{r-1}\right)\right] \stackrel{(a)}{\geq} 0 .
\end{aligned}
$$


The weak interference condition implies that for $r \in[1: i-1]$ :

$$
I\left(U ; Y_{r} \mid V X_{1, r}\right) \leq I\left(U ; Z_{k, r} \mid V X_{1, r}\right)
$$

where we let $U=Y_{i}$ and $V \equiv\left(Y^{r-1}, Z_{k, r+1}^{i-1}, W_{1}, X_{1,<r>}^{n}\right)$. Thus, letting $U_{i} \equiv\left(W_{1}, X_{1,<i>}, Y^{i-1}\right)$ completes the proof.

\section{REFERENCES}

[1] N. Devroye, P. Mitran, and V. Tarokh, "Achievable rates in cognitive radio channels," Information Theory, IEEE Transactions on, vol. 52, no. 5, pp. 1813-1827, 2006.

[2] I. Maric, R. Yates, and G. Kramer, "Capacity of interference channels with partial transmitter cooperation," Information Theory, IEEE Transactions on, vol. 53, no. 10, pp. 3536-3548, 2007.

[3] W. Wu, S. Vishwanath, and A. Arapostathis, "Capacity of a class of cognitive radio channels: Interference channels with degraded message sets," Information Theory, IEEE Transactions on, vol. 53, no. 11, pp. 4391-4399, 2007.

[4] A. Jovicic and P. Viswanath, "Cognitive radio: An information-theoretic perspective," Information Theory, IEEE Transactions on, vol. 55, no. 9, pp. 3945-3958, 2009.

[5] N. Liu, I. Maric, A. Goldsmith, and S. Shamai, "Bounds and capacity results for the cognitive z-interference channel," in Information Theory, 2009. ISIT 2009. IEEE Int. Sym. on, 2009, pp. 2422-2426.

[6] M. Vaezi, "The capacity of less noisy cognitive interference channels," in Communication, Control, and Computing (Allerton), 2012 50th Annual Allerton Conference on, 2012, pp. 1769-1774.

[7] —- "The capacity of more capable cognitive interference channels," CoRR, vol. abs/1207.2094, 2012. [Online]. Available: http://arxiv.org/abs/1207.2094

[8] S. Rini, D. Tuninetti, and N. Devroye, "New inner and outer bounds for the memoryless cognitive interference channel and some new capacity results," Information Theory, IEEE Transactions on, vol. 57, no. 7, pp. 4087-4109, 2011.

[9] — - "Inner and outer bounds for the gaussian cognitive interference channel and new capacity results," Information Theory, IEEE Transactions on, vol. 58, no. 2, pp. 820-848, 2012.

[10] J. Jiang, I. Maric, A. Goldsmith, S. Shamai, and S. Cui, "On the capacity of a class of cognitive z-interference channels," in Communications (ICC), 2011 IEEE International Conference on, 2011, pp. 1-6.

[11] M. Vaezi and M. Vu, "On the capacity of the cognitive z-interference channel," in Information Theory (CWIT), 2011 12th Canadian Workshop on, 2011, pp. 30-33.

[12] D. Maamari, D. Tuninetti, and N. Devroye, "Multi-user Cognitive Interference Channels: A Survey and New Capacity Results," ArXiv e-prints, Oct. 2015.

[13] M. Benammar and P. Piantanida, "On the compound broadcast channel: The role of multiple descriptions and interference decoding," submitted to IEEE Trans. Inf. Theor., 2014.

[14] A. Khisti, U. Erez, A. Lapidoth, and G. Wornell, "Carbon copying onto dirty paper," Information Theory, IEEE Transactions on, vol. 53, no. 5, pp. 1814-1827, 2007.

[15] M. Vaezi, "Comments on "new inner and outer bounds for the memoryless cognitive interference channel and some new capacity results"," Information Theory, IEEE Transactions on, vol. 59, no. 6, pp. 4055-4056, 2013.

[16] K. Marton, "A coding theorem for the discrete memoryless broadcast channel," Information Theory, IEEE Transactions on, vol. 25, no. 3, pp. 306-311, 1979. 
[17] T. Han and K. Kobayashi, "A new achievable rate region for the interference channel," Information Theory, IEEE Transactions on, vol. 27, no. 1, pp. 49-60, Jan 1981.

[18] C. Nair and Z. Wang, "The capacity region of the three receiver less noisy broadcast channel," Information Theory, IEEE Transactions on, vol. 57, no. 7, pp. 4058-4062, 2011.

[19] H. Weingarten, S. Shamai and G. Kramer, "On the compound MIMO broadcast channel," Information Theory and Applications (ITA 2007), UCSD, Palo-Alto, USA., Ed., Jan. 29-Feb. 2007.

[20] I. Csiszar and J. Krner, Information theory: coding theorems for discrete memoryless systems. Akadmiai Kiado, Budapest, 1982.

[21] T. Cover and J. Thomas, Elements of information theory (2nd Ed). Wiley-Interscience, 2006.

[22] I. Csiszar and J. Korner, "Broadcast channels with confidential messages," Information Theory, IEEE Transactions on, vol. 24, no. 3, pp. 339-348, 1978. 